\title{
Relaciones alométricas en estadios tempranos de la especie Brycon moorei Steindachner (Characidae), en condiciones controladas
}

\author{
Early-stage allometric relationships of the Brycon moorei Steindachner (Characidae) species, \\ under controlled conditions
}

Carlos A. David-Ruales ${ }^{1 *}$, Débora Machado-Fracalossi ${ }^{2}$, Eliana M. Betancur-Gonzalez ${ }^{1}$, Nicolás Rodríguez-Franco ${ }^{3}$, Germán Castañeda-Álvarez ${ }^{3}$, Cesar Florez-Restrepo ${ }^{1}$, Walter Vásquez-Torres ${ }^{4}$

\begin{abstract}
Resumen
Para Brycon moorei, los datos sobre morfometría, en estados iniciales del desarrollo, son inexistentes. El objetivo de este estudio fue establecer las principales relaciones alométricas en los estadios tempranos de B. moorei. Se analizaron 12 variables merísticas en un tiempo experimental que transcurrió desde la eclosión hasta los 15 días post-eclosión. En el estadio larval vitelino (LV) se registró, en promedio: longitud total (LT) de 4,74 \pm 0,05 mm; longitud estándar (LE) de 4,21 $\pm 0,07 \mathrm{~mm}$; longitud del hocico ( $\mathrm{SnL})$ 0,3 $\pm 0,01$; diámetro del ojo (ED) 0,37 $\pm 0,007 \mathrm{~mm}$; longitud de la cabeza (HL) 1,02 $\pm 0,04 \mathrm{~mm}$; altura de la cabeza $(\mathrm{HH}) 0,88$ $\pm 0,069 \mathrm{~mm}$; altura del cuerpo $(\mathrm{BH}) 1,26 \pm 0,01 \mathrm{~mm}$; longitud hocico-aleta pectoral (SnPF) $1,35 \pm 0,034$; ausencia de aletas pélvicas, anales y dorsal y el peso fue de 1,8 0,2 mg. En la etapa juvenil ( $\mathrm{J}$ ), las medidas fueron: LT de 25,66 \pm 0,64 mm; LE de 21,49 \pm 0,63 mm; SnL de 1,69 $\pm 0,059 \mathrm{~mm}$; ED de 1,71 $\pm 0,048 \mathrm{~mm}$; $\mathrm{HL}$ de 6,33 0,21 mm; HH de 4,89 $\pm 0,47 \mathrm{~mm}$; BH de 6,13 $\pm 0,17 \mathrm{~mm}$; SnPF de 6,48 0,19 mm; SnPeF de 10,83 \pm 0,28 mm; SnDF de 11,16 $\pm 0,33 \mathrm{~mm}$; Sn-AF de 3,68 $\pm 0,34 \mathrm{~mm}$; el peso fue de 221,6 $\pm 15,84$ mg. Para LV, el número de miómeros pre-anales fue $28,65 \pm 4,7$; los post-anales fueron $23,85 \pm 4,27$, con un total de 52,47 $\pm 8,34$; para J los miómeros no fueron visibles. Esta información es fundamental para fines ecológicos y productivos en $B$. moorei.
\end{abstract}

Palabras claves: alometría, larva, ontogenia

\begin{abstract}
For Brycon moorei, morphometry data in early stages of development is non-existent. The objective was to establish the main allometric relationships in the early stages of $B$. moorei. 12 meristic variables were analyzed in an experimental time ranging from hatching to 15 days post-hatching. In the larval vitelline stage (LV), it was recorded on average: total length (TL) of $4.74 \pm 0.05 \mathrm{~mm}$; standard length (EL) of $4.21 \pm 0.07$ $\mathrm{mm}$; snout length $(\mathrm{SnL}) 0.3 \pm 0.01$; eye diameter (ED) $0.37 \pm 0.007 \mathrm{~mm}$; head length (HL) $1.02 \pm 0.04 \mathrm{~mm}$; head height $(\mathrm{HH}) 0.88 \pm 0.069 \mathrm{~mm}$; body height $(\mathrm{BH}) 1.26 \pm 0.01 \mathrm{~mm}$; snout-pectoral fin length (SnPF) 1.35 \pm 0.034 ; absence of pelvic, anal and dorsal fins and the weight was $1.8 \pm 0.2 \mathrm{mg}$. In juvenile stage $(\mathrm{J})$, the measures were: LT of $25.66 \pm 0.64 \mathrm{~mm}$; LE of $21.49 \pm 0.63 \mathrm{~mm}$; SnL of $1.69 \pm 0.059 \mathrm{~mm} ; 1.71 \pm 0.048 \mathrm{~mm}$ ED; HL $6.33 \pm 0.21 \mathrm{~mm}$; HH of $4.89 \pm 0.47 \mathrm{~mm}$; BH of $6.13 \pm 0.17 \mathrm{~mm}$; SnPF of $6.48 \pm 0.19 \mathrm{~mm}$; SnPeF of $10.83 \pm 0.28 \mathrm{~mm}$; SnDF of $11.16 \pm 0.33 \mathrm{~mm}$; Sn-AF of $3.68 \pm 0.34 \mathrm{~mm}$; the weight was $221.6 \pm 15.84 \mathrm{mg}$. For the LV, the number of pre-anal myomers was $28.65 \pm 4.7$; the post-anal was $23.85 \pm 4.27$, with a total of $52.47 \pm 8.34$; for $\mathrm{J}$ the myomers were not visible. This information is essential for ecological and productive purposes in B. moorei.
\end{abstract}

Keywords: allometry, larvae, ontogeny

\footnotetext{
1. Corporación Universitaria Lasallista, Caldas, Colombia.

2. Laboratorio de Nutrición de Peces (LABNUTRI), Universidad Federal de Santa Catarina, Santa Catarina, Brasil.

3. Piscícola Doradal, Puerto Triunfo, Colombia.

4. Instituto de Acuicultura de los Llanos (IALL), Universidad de los Llanos, Villavicencio, Colombia.

* Autor de correspondencia: <cadavid@lasallistadocentes.edu.co>
} 


\section{INTRODUCCIÓN}

La ontogenia de la mayoría de las larvas de peces se caracteriza por cambios drásticos que determinan su capacidad fisiológica y de comportamiento durante el desarrollo (Peña, y Dumas, 2009). Los cambios funcionales y corporales de las larvas en los primeros días después de la eclosión, están relacionados con patrones característicos de crecimiento alométrico y su cronología se asocia con importantes eventos en su historia de vida (Gisbert, 1999). El análisis del crecimiento de las larvas, en etapas iniciales de desarrollo, proporciona información sobre su comportamiento y ecología y puede utilizarse para evaluar relaciones filogenéticas (Gisbert, y Doroshov, 2006). Se conoce que existen variaciones interespecíficas en los tiempos de desarrollo y funcionabilidad, por lo que es importante orientar estudios de crecimiento para optimizar las técnicas de cría de larvas y de condiciones de alimentación (Padrós, Villalta, Gisbert, y Estévez, 2011); así, el crecimiento de una especie está determinado por la respuesta celular a varios factores internos y externos, como se ha observado en larvas de peces y anfibios (Mansano, De Stéfani, Pereira, Nascimento, y Macente, 2014; Mansano et al, 2017). Al respecto, la explicación del crecimiento en larvas de peces y anfibios usa modelos matemáticos que pretenden hacer una descripción, control y predicción de las variables asociadas (peso, talla, entre otras) (Mansano et al, 2017). Los patrones de crecimiento alométrico son los más comunes en larvas de peces (Fuiman, 1983) y representan prioridades en sus funciones vitales (Osse y van den Boogaart, 1995; Osse, van den Boogaart, van Snik, y van der Sluys, 1997). Para larvas de peces de agua dulce los reportes de Bettinelli-Nogueira, Lima-Godinho, y PereiraGodinho (2014), Oliveira, Bialetzki, Gomes, Santin, y Taguti (2012); Souza, Melo, Caramaschi, Andrade, y Monteiro (2016) y Souza, Caramaschi, y Monteiro (2017), sustentan lo descrito anteriormente.

De manera general, la historia de vida de los peces puede ser malinterpretada si únicamente se consideran, para su estudio, sus formas adultas (Balon, 1981, 1986). Además, pequeñas fallas en la identificación de larvas de peces pueden generar grandes errores en la interpretación de fenómenos ecológicos y taxonómicos, en vista de que el estado larval puede tener diferentes funciones ecológicas en diferentes animales (Orton, 1953). Por lo tanto, considerar el desarrollo temprano en las larvas puede anexar detalles que ayudan a validar su identificación (Powles y Markle, 1983). Se sabe que la anatomía larval es dinámica y que usualmente posee pocas tipologías asociadas a los adultos, haciendo su identificación más compleja; para ello, estrategias como las de reproducir en ambientes controlados u obtener huevos y larvas de padres conocidos es una aproximación válida para la identificación de estadios tempranos de desarrollo (Leiby, 1984).

Todos estos cambios, regulados por la relación ambiente-genotipo (Gilbert, y Bolker, 2003), determinan fenotipos que varían en sus coeficientes de crecimiento, cambiando sus proporciones alométricas (Gisbert, y Doroshov, 2006) y la forma cómo las larvas aprovechan el ambiente (Devlin, Vandersteen, Uh, y Stevens, 2012). De esta manera, la característica más importante para la identificación del desarrollo temprano es la merística, ya que puede ser usada a diferentes niveles taxonómicos (Kendall, Ahlstrom, y Moser, 1983).

La dorada Brycon moorei, es una especie en estado de vulnerabilidad (categoría A2c,d -Nacional en Peligro Crítico (A2c) - Regional) por la reducción de su tamaño poblacional en los últimos años (Mojica, Usma, Álvarez-León, y Lasso, 2012); además, no se encuentran reportes sobre su morfología, patrones de crecimiento y relaciones corporales. Por lo tanto, el objetivo del presente trabajo fue realizar el seguimiento morfométrico completo y las relaciones merísticas en su desarrollo temprano para sentar las bases de su gestión y conservación en el medio natural, así como también para su manejo en acuicultura.

\section{MATERIALES Y MÉTODOS}

\section{Localización}

La fase experimental, con peces vivos, se desarrolló en la Estación Piscícola Doradal localizada a 165 $\mathrm{km}$ de Medellín, en el corregimiento de Doradal, Municipio de Sonsón, Antioquia, Colombia; a una altitud de $150 \mathrm{~m}$ s. n. m. 


\section{Plantel de reproductores, mantenimiento en cautiverio, eventos reproductivos y desarrollo embrionario}

Reproductores de dorada de un año de edad del río Magdalena, completamente adaptados al confinamiento, fueron alimentados con una dieta comercial del $38 \%$ de proteína cruda (PC) para tilapia (Oreochromis sp.), cuya composición nutricional fue $38 \%$ de PC mínimo; grasa mínima 4\%; cenizas máximas $12 \%$; humedad máxima $13 \%$ y fibra máxima $4 \%$. El registro y la evaluación de los eventos reproductivos se realizaron según David-Ruales y CastañedaÁlvarez, (2014). El seguimiento del desarrollo embrionario hasta el momento de la eclosión de la larva se hizo, con algunas modificaciones, según lo reportado por Kimmel, Ballard, Kimmel, Ullmann, y Schilling (1995) y Tsai, Chang, Liu, Abe, y Ota (2013). De los huevos fecundados se obtuvieron las variables: diámetro total del huevo (ED), diámetro del vitelo (YD) y espacio perivitelino (PS), el cual fue caracterizado de acuerdo con su participación en el volumen total del huevo (Nakatani, Agostinho, Baumgartner, Bialetzki, Sanches y Cavicchioli, 2001) (figura 1A y 1B).

\section{Larvicultura, alevinaje, protocolo alimenticio y recolección de muestras}

Se realizaron en un sistema de recirculación, con 25 tanques de $60 \mathrm{~L}$ de volumen efectivo, unidos a un sistema de filtración con desgasificador, filtro biomecánico y lámpara UV, el cual mantuvo los parámetros ambientales dentro de rangos de confort de la especie: OD $\left(5,5 \pm 0,5 \mathrm{mg} \mathrm{L}^{-1}\right), \mathrm{pH}(6,5 \pm$ $0,8)$, temperatura en ${ }^{\circ} \mathrm{C}(26 \pm 0,4)$, alcalinidad $\mathrm{y}$ dureza $\left(20,8 \pm 1,7 \mathrm{mg} \mathrm{L}^{-1} \mathrm{CaCO}_{3}\right)$ y $\mathrm{NH}_{3}(0,002$ $\left.\pm 0,00001 \mathrm{mg} \mathrm{L}^{-1}\right)$. El registro de los parámetros anteriores de calidad del agua se realizó una vez al día a las 7:00 am, con un equipo medidor de calidad de agua (Kit Hach FF2) y con una sonda multiparamétrica YSI (Profesional plus). El caudal de entrada de cada tanque fue controlado por medio de flujómetros (Parker MR) y se mantuvo en $3,5 \mathrm{~L}$ $\mathrm{min}^{-1}$; las demás características ambientales y de manejo del sistema se realizaron según David-Ruales, y Castañeda-Álvarez (2014). Para ello, a las larvas de 26 h post eclosión (HPE) (17 Larvas $\mathrm{L}^{-1}$ ), se les realizaron biometrías cada $3 \mathrm{~h}$ hasta las $47 \mathrm{HPE}$; cada $4 \mathrm{~h}$ hasta las $71 \mathrm{HPE}$; cada 6 h hasta las 95 HPE; cada 8 h hasta las $111 \mathrm{HPE}$; cada 12 h hasta las 135 HPE y una muestra diaria hasta el día 15 (Faustino, 2010; Neumann, 2008). A todas las larvas se les aplicó un protocolo alimenticio ad libitum que en los tres primeros días incluyó larvas forrajeras de bocachico (Prochilodus magdalenae), luego una combinación con Artemia salina y, a partir del sexto día, una ración con alimento balanceado del $45 \%$ de PC, que incrementó hasta el día 15; la tabla 1 ilustra la técnica utilizada para la alimentación de las larvas.

Tabla 1. Protocolos de alimentación para larvas de Brycon moorei.

\begin{tabular}{c|c|c|c|c|c}
\hline \multirow{2}{*}{ DPE } & \multicolumn{5}{|c}{ HORAS DE ALIMENTACIÓN } \\
& $\mathbf{7}$ & $\mathbf{1 0}$ & $\mathbf{1 3}$ & $\mathbf{1 6}$ & $\mathbf{1 9}$ \\
\hline 1 & $\mathrm{LF}$ & $\mathrm{LF}$ & $\mathrm{LF}$ & $\mathrm{LF}$ & $\mathrm{LF}$ \\
2 & $\mathrm{LF}$ & $\mathrm{LF}$ & $\mathrm{LF}$ & $\mathrm{LF}$ & $\mathrm{LF}$ \\
3 & $\mathrm{LF}$ & $\mathrm{LF}$ & $\mathrm{LF}$ & $\mathrm{LF}$ & $\mathrm{LF}$ \\
4 & $\mathrm{~A}$ & $\mathrm{~A}$ & $\mathrm{~A}$ & $\mathrm{~A}$ & $\mathrm{~A}$ \\
5 & $\mathrm{~A}$ & $\mathrm{~A}$ & $\mathrm{~A}$ & $\mathrm{~A}$ & $\mathrm{~A}$ \\
6 & $\mathrm{~A}$ & $\mathrm{~A}$ & $\mathrm{~A}+\mathrm{C}$ & $\mathrm{A}$ & $\mathrm{A}$ \\
7 & $\mathrm{~A}$ & $\mathrm{~A}$ & $\mathrm{~A}+\mathrm{C}$ & $\mathrm{A}$ & $\mathrm{A}$ \\
8 & $\mathrm{~A}$ & $\mathrm{~A}$ & $\mathrm{~A}+\mathrm{C}$ & $\mathrm{A}$ & $\mathrm{A}$ \\
9 & $\mathrm{~A}+\mathrm{C}$ & $\mathrm{A}$ & $\mathrm{A}$ & $\mathrm{C}$ & $\mathrm{A}+\mathrm{C}$ \\
10 & $\mathrm{~A}+\mathrm{C}$ & $\mathrm{C}$ & $\mathrm{C}$ & $\mathrm{A}+\mathrm{C}$ & $\mathrm{C}$ \\
11 & $\mathrm{C}$ & $\mathrm{C}$ & $\mathrm{A}+\mathrm{C}$ & $\mathrm{C}$ & $\mathrm{C}$ \\
12 & $\mathrm{~A}+\mathrm{C}$ & $\mathrm{C}$ & $\mathrm{C}$ & $\mathrm{C}$ & $\mathrm{C}$ \\
13 & $\mathrm{C}$ & $\mathrm{C}$ & $\mathrm{C}$ & $\mathrm{C}$ & $\mathrm{C}$ \\
14 & $\mathrm{C}$ & $\mathrm{C}$ & $\mathrm{C}$ & $\mathrm{C}$ & $\mathrm{C}$ \\
15 & $\mathrm{C}$ & $\mathrm{C}$ & $\mathrm{C}$ & $\mathrm{C}$ & $\mathrm{C}$ \\
\hline
\end{tabular}

LF: larva forrajera de Prochilodus magdalenae; A: nauplios de Artemia salina; C: concentrado del $45 \%$ de PC; DPE: días post-eclosión.

Las larvas recolectadas (10) se clasificaron con base en lo descrito por Kendall et al (1983) y modificado por Nakatani et al (2001). El grado de flexión de la notocorda y el desarrollo de estructuras como la aleta caudal y las escamas, entre otras, determinaron siete etapas: 1. Larval Vitelino (LV), etapa entre la eclosión y el inicio de la alimentación exógena; 2. PreFlexión (PF), etapa entre el inicio de la alimentación exógena, hasta el inicio de la flexión de la región terminal de la notocorda; 3. Inicio Flexión (iF), etapa intermedia del inicio de la flexión de la notocorda; 4 . Flexión $(\mathrm{F})$, etapa donde aparece la aleta pélvica y radios de la aleta caudal; se observa claramente la 


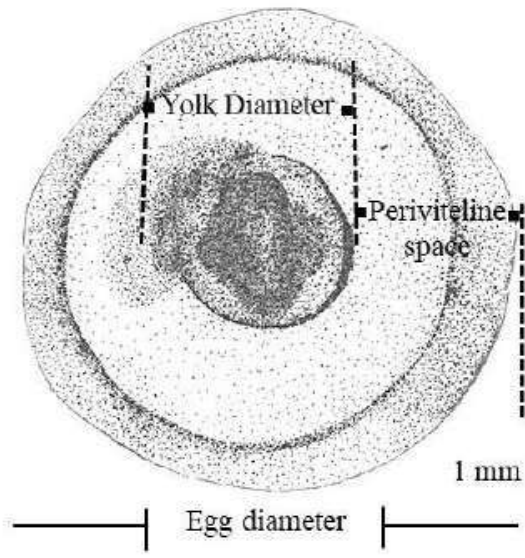

Figura $1 \mathrm{~A}$

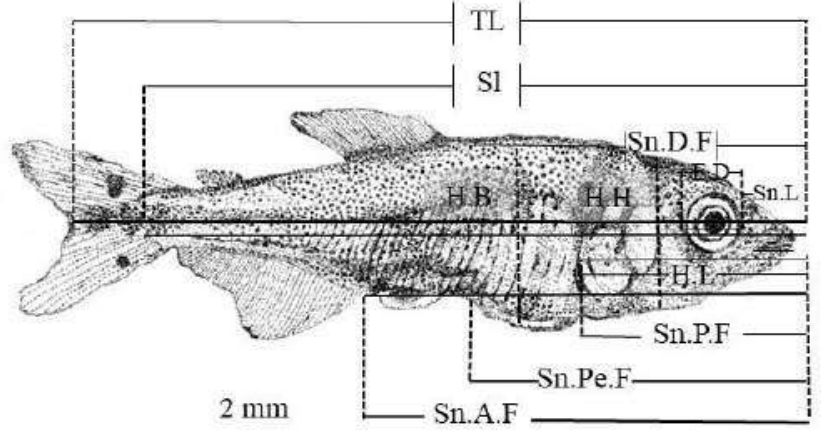

Figura 1B

Figura 1. Diagrama de las medidas tomadas: A. huevo. B. juveniles de Brycon moorei. ED: diámetro total del huevo, YD: diámetro del saco de vitelo, PS: espacio perivitelino, TL: longitud total, SL: longitud estándar, Sn.L: longitud hocico, ED: diámetro ojo, HL: longitud cabeza, HH: altura cabeza, BH: altura del cuerpo, SnDF: distancia hocico a aleta dorsal, SnPF: distancia hocico a aleta pectoral, SnPeF: distancia hocico a aleta pélvica, SnAF: distancia hocico a aleta anal.

flexión de la notocorda; 5. Inicio Post-Flexión (iPtF) - 6. Post-Flexión (PtF), marcados por la flexión total de la notocorda, formación completa de huesos hipurales y 7 . Juvenil (J), etapa donde se observa la formación total de los radios de las aletas, aparición de escamas y absorción total de la aleta primigenia. Todas las larvas se fijaron en formol tamponado al $10 \%$ y fueron observadas en un estereomicroscopio SteREO Discovery.V12 (Carl Zeiss Microscopy, LLC) con analizador de imagen.

\section{Análisis morfométrico}

Las variables morfométricas para larvas y juveniles fueron: longitud total (TL), longitud estándar (SL), longitud del hocico (Sn.L), diámetro del ojo (ED), longitud de la cabeza (HL), altura de la cabeza $(\mathrm{HH})$, longitud del hocico a la aleta dorsal (Sn.D.F), longitud del hocico a la aleta pectoral (Sn.P.F), longitud del hocico a la aleta pélvica (Sn.Pe.F), longitud del hocico a la aleta anal (Sn.A.F) y altura del cuerpo (HB); la figura 1-b, muestra específicamente estas medidas para juveniles. Las variables merísticas también abarcaron el número total, el número pre-anal y post-anal de miómeros.
Las medidas corporales fueron transformadas a logaritmo en base 10, ajustando la ecuación alométrica $\mathrm{Y}=\mathrm{aX}^{b}$ (Fuiman, 1983), la cual pasó a ser, después de la transformación, a la ecuación $\boldsymbol{L}_{\boldsymbol{o g}} \boldsymbol{Y}=\boldsymbol{L}_{10} \boldsymbol{g}_{10} \boldsymbol{a}$ $+\boldsymbol{b L o g}_{10} \boldsymbol{X}$ (Stewart, 2008), permitiendo mejorar el coeficiente de determinación $\left(\mathrm{R}^{2}\right)$, arrojando un modelo más confiable para pronósticos en nuevas observaciones y análisis de máximos y mínimos de las variables relacionadas; siendo $\boldsymbol{Y}$ la variable dependiente, $\boldsymbol{X}$ la variable independiente, $\boldsymbol{a}$ el valor del intercepto en logaritmo y $\boldsymbol{b}$, el valor de la pendiente o coeficiente alométrico (Fuiman, 1983). Los conceptos de isometría y alometría basados en el valor de la pendiente dan como resultado, si $\boldsymbol{b}=\mathbf{1}, \mathrm{Y}$ y X, crecen a la misma velocidad (isometría); si $\boldsymbol{b}>\mathbf{1}, \mathrm{Y}$ crece más rápido que $\mathrm{X}$ (alometría positiva) y si $b<1$, $\mathrm{Y}$ crece más despacio que $\mathrm{X}$ (alometría negativa) (Peña, y Dumas, 2009). Para evaluar el crecimiento en todo el ciclo experimental, los valores de longitud total y estándar y su relación con respecto al tiempo en HPE se ajustaron al modelo alométrico transformado a logaritmo como fue descrito previamente $\left(\log _{10} Y=\log _{10} a+b \log _{10} X\right)$. Para obtener los intervalos de confianza al $95 \%$, se usó el estadístico "t-student", con n-2 grados de libertad, para la pen- 
diente (coeficiente de alometría) del modelo transformado (Walpole, Myers, Myers, y Ye, 2012). Las relaciones establecidas fueron: longitud total (LT) (X) y longitud estándar (LE) (Y), LE (X) y altura de la cabeza $(\mathrm{HCBz})(\mathrm{Y})$, LE (X) y longitud cabeza $(\mathrm{LCBz})(\mathrm{Y}), \mathrm{LE}(\mathrm{X})$ y altura del cuerpo (H-Cuerpo) (Y) y $\mathrm{LCBz}(\mathrm{X})$ y diámetro del ojo (Diam.OJO)(Y). Cada relación se representó en un diagrama de dispersión, ajustando los datos a la línea recta con base en la ecuación alométrica descrita anteriormente (Ricker, 1979). El desempeño en crecimiento para la especie fue evaluado con base en la Tasa de Crecimiento Instantáneo (TCI), de acuerdo con la fórmula $T C I=\left(L n L T_{2}-L n L_{1}\right) / t_{2}-t_{1}$; siendo $\operatorname{LnLT}_{2} \mathrm{y}$ $\mathrm{LnLT}_{1}$ los logaritmos naturales de los promedios de las longitudes totales en sus respectivos tiempos $\left(t_{2}\right.$ y $\left.t_{1}\right)$ (Ricker, 1979). Todos los datos se analizaron con el paquete estadístico Stat-Graphics Centurion $\mathrm{XV}$, con licencia de la Corporación Universitaria Lasallista.

\section{RESULTADOS}

\section{Periodo embrionario}

Presenta una duración de 13 h que van desde la fertilización hasta la eclosión. En 190 huevos se midió diámetro total del huevo (ED), diámetro del vitelo (YD) y espacio perivitelino (PS), desde los $90 \mathrm{~s}$ hasta la $4^{a}$ hora después de la fertilización. ED obtuvo un promedio de 2,72 $\pm 0,12 \mathrm{~mm}$ ( \pm desviación estándar), con un mínimo de 2,077 mm y un máximo de $3,21 \mathrm{~mm}$; YD, presentó una longitud promedio de $1,44 \pm 0,05 \mathrm{~mm}$, con un valor mínimo de $0,76 \mathrm{~mm}$ y un máximo de 1,80 mm; PS, con relación al volumen total del huevo, fue "restricto" $(3,73 \%)$ y "moderado" con respecto a $\mathrm{ED}(15,4 \%)$, con un promedio de 0,42 $\pm 0,05 \mathrm{~mm}$, un valor mínimo de $0,12 \mathrm{~mm}$ y un valor máximo de 0,72 mm. El embrión midió 2,24 $\pm 0,21$ $\mathrm{mm}$ y su saco vitelino $0,51 \pm 0,1 \mathrm{~mm}^{3}$. La tabla 2 resume los valores encontrados en esta etapa.

\section{Periodo larval}

La larva eclosiona a las 12 h post-fertilización; este periodo va desde las $0 \mathrm{~h}$ post-eclosión (HPE) hasta la $254 \mathrm{HPE}$, que corresponde desde larval vitelino (LV) hasta Post-Flexión (PtF). La figura 2, describe las etapas de desarrollo larval, comprendidas entre las 0 HPE hasta los 15 días post-eclosión (DPE); la tabla 2 indica todas las medidas y sus relaciones desde LV hasta juvenil $(\mathrm{J})$.

\section{Estadio larval vitelino (LV) (figura 2A y 2B-tabla 2)}

Periodo de tiempo comprendido entre la hora 13 post-fertilización (HPF), hasta las $24 \mathrm{HPE}$. Las larvas alcanzaron valores promedios en TL de 4,75 mm; SL de 4,21 mm; ED de 0,37 mm; BH de 1,26, HL de $1,02 \mathrm{~mm}$ y $\mathrm{HH}$ de $0,88 \mathrm{~mm}$. La relación $\mathrm{ED} / \mathrm{HL}$ del 34,89 $\pm 0,63 \%$, indica un ojo grande; HL/SL del $24,06 \pm 0,37 \%$ y BH/SL del 30,91 $\pm 0,26$, indican respectivamente una longitud de cabeza y una longitud del cuerpo moderados. Al eclosionar la larva ya presenta un ojo en su mayoría pigmentado y algunos melanocitos dendríticos en la parte anterior del saco vitelino, patrón de pigmentación que incrementa durante toda la fase larval. Hacia la $8 \mathrm{HPE}$, el brote de la aleta pectoral comienza a girar hacia la parte posterior. El peso promedio en esta etapa fue 1,8 \pm 0,2 mg.

\section{Estadio pre-flexión (PF) (figura 2C-tabla 2)}

Periodo de tiempo comprendido entre las 25 hasta las 53 HPE. Las larvas alcanzaron valores promedios en TL de $6,85 \mathrm{~mm}$, incremento que corresponde al $44 \%$ con relación a la etapa anterior; SL de 6,32 mm; ED de 0,39 mm; BH de 1,19, el cual redujo su crecimiento en un 5,6\% debido a la reducción del vitelo; HL de 1,29 mm y $\mathrm{HH}$ de 1,18 $\mathrm{mm}$. La relación ED/HL del 30,41 $\pm 0,18 \%$, indica un ojo moderado; HL/SL del 20,3 $\pm 0,74 \%$ y BH/SL del 18,95 \pm 0,79 , indican respectivamente una longitud de cabeza moderada y una longitud del cuerpo largo. Aún se observan vestigios de la aleta primigenia; la vejiga gaseosa a las $44 \mathrm{HPE}$ se ve inflada, transparente y externamente pigmentada en su región dorsal; los patrones de pigmentación en general incrementan en la región ventral y cefálica; el opérculo cubre toda la abertura branquial y llega hasta el brote de la aleta pectoral. 


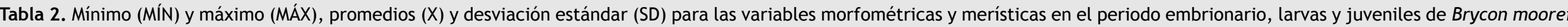
(adaptada de Nakatani et al, 2001).

\begin{tabular}{|c|c|c|c|c|c|c|c|c|c|c|c|c|c|c|}
\hline \multirow{4}{*}{$\begin{array}{l}\text { VARIABLES } \\
(\mathrm{mm}-\mathrm{mg})\end{array}$} & \multicolumn{12}{|c|}{ LARVAL } & \multicolumn{2}{|c|}{ JUVENIL } \\
\hline & \multirow{2}{*}{\multicolumn{2}{|c|}{$\begin{array}{c}\text { LV }(n=180) \\
0-24 \text { HPE }\end{array}$}} & \multirow{2}{*}{\multicolumn{2}{|c|}{$\begin{array}{c}\text { PF }(n=90) \\
25-53 \text { HPE } \\
(38-67 \text { HPF })\end{array}$}} & \multirow{2}{*}{\multicolumn{2}{|c|}{$\begin{array}{c}\text { iF }(n=50) \\
56-71 \text { HPE } \\
(69-84 \mathrm{HPF})\end{array}$}} & \multirow{2}{*}{\multicolumn{2}{|c|}{$\begin{array}{c}F(n=50) \\
82-110 \mathrm{HPE} \\
(95-123 \mathrm{HPF})\end{array}$}} & \multirow{2}{*}{\multicolumn{2}{|c|}{$\begin{array}{c}\text { iPtF }(n=40) \\
123-182 \mathrm{HPE} \\
(136-195 \mathrm{HPF})\end{array}$}} & \multirow{2}{*}{\multicolumn{2}{|c|}{$\begin{array}{c}\text { PtF }(n=30) \\
206-254 \text { HPE } \\
(219-267 H P F)\end{array}$}} & \multirow{2}{*}{\multicolumn{2}{|c|}{$\begin{array}{c}J(n=30) \\
278-326 \text { HPE } \\
(291-339 H P F)\end{array}$}} \\
\hline & & & & & & & & & & & & & & \\
\hline & $\mathrm{X} \pm \mathrm{SD}$ & MÍN-MÁX & $\mathbf{X} \pm \mathbf{S D}$ & MÍN-MÁX & $\mathrm{X} \pm \mathrm{SD}$ & MÍN-MÁX & $\mathrm{X} \pm \mathrm{SD}$ & MÍN-MÁX & $\mathrm{X} \pm \mathrm{SD}$ & MÍN-MÁX & $\mathrm{X} \pm \mathrm{SD}$ & MÍN-MÁX & $\mathrm{X} \pm \mathrm{SD}$ & MÍN-MÁX \\
\hline$\overline{T L}$ & $4,74 \pm 0,05$ & $3,05-5,66$ & $6,85 \pm 0,53$ & $6,22-7,48$ & $7,89 \pm 0,14$ & $7,39-8,12$ & $9,85 \pm 0,09$ & $8,9-10,73$ & $13,81 \pm 0,12$ & $11,38-16,31$ & $15,82 \pm 0,41$ & $14,32-18,22$ & $25,66 \pm 0,64$ & $24,45-26,01$ \\
\hline SL & $4,21 \pm 0,06$ & $2,68-5,14$ & $6,32 \pm 0,099$ & $5,83-6,69$ & $7,35 \pm 0,13$ & $6,98-7,6$ & $9,26 \pm 0,07$ & $8,34-10,14$ & $12,44 \pm 0,13$ & $10,48-14,5$ & $13,68 \pm 0,049$ & $12,25-15,69$ & $21,49 \pm 0,33$ & $21,35-21,56$ \\
\hline Sn.L & $0,3 \pm 0,01$ & $0,29-0,33$ & $0,42 \pm 0,02$ & $0,39-0,46$ & $0,49 \pm 0,021$ & $0,44-0,53$ & $0,63 \pm 0,021$ & $0,58-0,69$ & $0,95 \pm 0,026$ & $0,7-1,15$ & $1,13 \pm 0,049$ & $1,04-1,29$ & $1,69 \pm 0,059$ & $1,34-1,93$ \\
\hline ED & $0,37 \pm 0,007$ & $0,16-0,45$ & $0,39 \pm 0,0042$ & $0,353-0,41$ & $0,46 \pm 0,01$ & 0,43-49 & $0,66 \pm 0,005$ & $0,59-0,75$ & $0,96 \pm 0,012$ & $0,79-1,15$ & $1,10 \pm 0,025$ & $1,02-1,22$ & $1,71 \pm 0,048$ & $1,62-1,85$ \\
\hline HL & $1,02 \pm 0,04$ & $0,53-1,38$ & $1,29 \pm 0,037$ & $1,16-1,45$ & $1,32 \pm 0,051$ & $1,2-1,499$ & $1,96 \pm 0,05$ & $1,84-2,29$ & $3,24 \pm 0,039$ & $2,11-4,14$ & $3,47 \pm 0,064$ & $3,19-4,33$ & $6,33 \pm 0,21$ & $5,82-6,55$ \\
\hline $\mathrm{HH}$ & $0,88 \pm 0,069$ & $0,47-1,23$ & $1,18 \pm 0,04$ & $1,009-1,39$ & $1,37 \pm 0,01$ & $1,25-1,45$ & $1,82 \pm 0,017$ & $1,62-2,05$ & $2,65 \pm 0,11$ & $1,97-3,3$ & $3,29 \pm 0,16$ & $2,72-3,68$ & $4,89 \pm 0,47$ & $4,3-5,43$ \\
\hline $\mathrm{BH}$ & $1,26 \pm 0,01$ & $1,22-1,4$ & $1,19 \pm 0,053$ & $1,07-1,29$ & $1,45 \pm 0,097$ & $1,27-1,55$ & $2,12 \pm 0,04$ & $1,904-2,33$ & $3,13 \pm 0,053$ & $2,12-4,03$ & $3,6 \pm 0,065$ & $2,77-4,42$ & $6,13 \pm 0,17$ & $4,97-6,8$ \\
\hline Sn-PF & $1,35 \pm 0,044$ & $1-1,68$ & $1,71 \pm 0,055$ & $1,602-1,808$ & $1,86 \pm 0,021$ & $1,76-1,92$ & $2,33 \pm 0,037$ & $2,11-2,57$ & $3,67 \pm 0,12$ & $2,68-4,40$ & $4,27 \pm 0,13$ & $3,78-4,93$ & $6,48 \pm 0,19$ & $5,75-6,92$ \\
\hline Sn-PeF & AAU & AAU & AAU & AAU & AAU & AAU & AAU & AAU & $7,15 \pm 0,15$ & $7,08-9,68$ & $8,17 \pm 0,17$ & 7-9,07 & $10,83 \pm 0,28$ & $9,09-11,9$ \\
\hline Sn-DF & AAU & AAU & AAU & AAU & AAU & AAU & $5,47 \pm 0,12$ & $4,22-6,25$ & $7,12 \pm 0,10$ & $6,07-8,47$ & $7,742 \pm 0,18$ & $6,89-8,81$ & $11,16 \pm 0,33$ & $9,77-12,16$ \\
\hline Sn-AF & AAU & AAU & AAU & AAU & AAU & AAU & $6,67 \pm 0,11$ & $6,01-7,18$ & $8,7 \pm 0,085$ & $7,3-10,06$ & $9,49 \pm 0,17$ & $8,48-10,67$ & $13,78 \pm 0,34$ & $12,08-14,86$ \\
\hline Peso (mg) & $1,8 \pm 0,2$ & $0,8-4,13$ & $3,14 \pm 0,36$ & $2,5-4,27$ & $4,37 \pm 0,27$ & $3,24-5,63$ & $10,92 \pm 0,57$ & $7,96-14,41$ & $32,14 \pm 3,55$ & $15,28-52,79$ & $60,90 \pm 10,01$ & $37,13-74,71$ & $221,6 \pm 15,84$ & $\begin{array}{l}213,58- \\
225,79\end{array}$ \\
\hline \multicolumn{15}{|c|}{ NÚMERO DE MIÓMEROS } \\
\hline Pré-Anal & $28,65 \pm 4,7$ & $23-33$ & $31,8 \pm 1,86$ & $28-36$ & $31,4 \pm 1,02$ & $30-33$ & $31,4 \pm 1,14$ & $30-33$ & NV & NV & NV & NV & NV & NV \\
\hline Póst-Anal & $23,85 \pm 4,27$ & $12-32$ & $28,73 \pm 2,28$ & $25-32$ & $28,2 \pm 1,2$ & $26-29$ & $28,2 \pm 1,3$ & $26-29$ & NV & NV & NV & NV & NV & NV \\
\hline Total & $52,47 \pm 8,34$ & $33-66$ & $60,53 \pm 3,13$ & $53-68$ & $59,6 \pm 1,05$ & $58-61$ & $59,6 \pm 1,14$ & $58-61$ & NV & NV & NV & NV & NV & NV \\
\hline \multicolumn{15}{|c|}{ NÚMERO DE RADIOS } \\
\hline CF & & & & & & & & & & & $24,66 \pm 0,57$ & $24-25$ & $26 \pm 0,7$ & $25-27$ \\
\hline DF & & & & & & & & & & & $9,2 \pm 0,44$ & $9-10$ & $12 \pm 0,7$ & $11-13$ \\
\hline PF & & & & & & & & & & & NV & NV & $15,33 \pm 0,57$ & $15-16$ \\
\hline PeF & & & & & & & & & & & NV & NV & $9,6 \pm 0,54$ & $9-10$ \\
\hline $\mathrm{AF}$ & & & & & & & & & & & $20,5 \pm 1$ & $20-22$ & $30,57 \pm 0,97$ & 29-32 \\
\hline \multicolumn{15}{|c|}{ PROPORCIONES CORPORALES (\%) } \\
\hline $\mathrm{ED} / \mathrm{HL}$ & $35,89 \pm 0,63$ & $30,64-47,24$ & $30,41 \pm 0,18$ & $28,25-33,55$ & $35,67 \pm 0,36$ & $\begin{array}{c}31,014- \\
38,22\end{array}$ & $34,2 \pm 0,24$ & $\begin{array}{l}31,99- \\
36,43\end{array}$ & $30,55 \pm 0,21$ & $27,62-37,68$ & $29,91 \pm 0,087$ & $\begin{array}{l}28,12- \\
32,067\end{array}$ & $27,17 \pm 0,061$ & $25,7-27,96$ \\
\hline $\mathrm{HL} / \mathrm{SL}$ & $24,06 \pm 0,37$ & $18,91-28,67$ & $20,3 \pm 0,74$ & $18,01-22,19$ & $17,85 \pm 0,3$ & $\begin{array}{l}16,61- \\
20,16\end{array}$ & $21,14 \pm 0,36$ & $\begin{array}{l}19,97- \\
22,59\end{array}$ & $25,76 \pm 0,38$ & $20,14-28,60$ & $27,28 \pm 0,43$ & $26,05-28,14$ & $29,45 \pm 0,095$ & $27-30,69$ \\
\hline $\mathrm{HH} / \mathrm{SL}$ & $21 \pm 0,05$ & $11,08-29,2$ & $18,67 \pm 0,069$ & $15,96-22$ & $18,64 \pm 0,09$ & $17-19,7$ & $19,65 \pm 0,043$ & $17,49-22,1$ & $21,3 \pm 0,175$ & $15,8-26,5$ & $24,04 \pm 0,1$ & $19,9-26,9$ & $22,75 \pm 0,4$ & $20-25,26$ \\
\hline $\mathrm{BH} / \mathrm{SL}$ & $30,91 \pm 0,26$ & $23,89-50,75$ & $18,95 \pm 0,79$ & $16,44-20,88$ & $19,74 \pm 0,6$ & $\begin{array}{l}18,25- \\
21,03\end{array}$ & $22,94 \pm 0,31$ & $\begin{array}{c}22,47- \\
23,59\end{array}$ & $24,91 \pm 0,4$ & $20,27-27,78$ & $28,62 \pm 0,57$ & $\begin{array}{l}27,57- \\
30,094\end{array}$ & $28,56 \pm 0,082$ & $23,06-31,54$ \\
\hline YL/SL & $31,27 \pm 0,07$ & $29,74-33,19$ & $15,83 \pm 0,13$ & $4,21-19,85$ & S.D & S.D & S.D & S.D & S.D & S.D & S.D & S.D & S.D & S.D \\
\hline $\mathrm{YH} / \mathrm{SL}$ & $19,35 \pm 0,05$ & $17,9-21,4$ & $9,08 \pm 0,09$ & $1,57-12,73$ & S.D & S.D & S.D & S.D & S.D & S.D & S.D & S.D & S.D & S.D \\
\hline Sn-PF/SL & $32,06 \pm 0,082$ & $23,75-39,9$ & $27,05 \pm 0,07$ & $25,34-28,6$ & $25,3 \pm 0,07$ & $\begin{array}{l}23,94- \\
26,12\end{array}$ & $25,16 \pm 0,05$ & $22,78-27,7$ & $29,5 \pm 0,12$ & $21,54-35,36$ & $31,21 \pm 0,15$ & $27,63-36,03$ & $30,15 \pm 0,35$ & $26,75-32,2$ \\
\hline $\begin{array}{c}\text { Sn- } \\
\text { PeF/SL }\end{array}$ & S.D & S.D & S.D & S.D & S.D & S.D & S.D & S.D & $57,47 \pm 0,14$ & $56,91-77,81$ & $59,72 \pm 0,1$ & $51,16-66,3$ & $50,39 \pm 0,3$ & $42,29 \pm 55,37$ \\
\hline Sn-DF/SL & S.D & S.D & S.D & S.D & S.D & S.D & $59,07 \pm 0,09$ & $\begin{array}{c}45,57- \\
67,49\end{array}$ & $57,23 \pm 0,11$ & $48,79-68,08$ & $56,59 \pm 0,11$ & $50,36-64,4$ & $51,93 \pm 0,33$ & $45,46-56,58$ \\
\hline Sn-AF/SL & S.D & S.D & S.D & S.D & S.D & S.D & $72,03 \pm 0,09$ & $64,9-77,53$ & $69,93 \pm 0,1$ & $58,68-80,86$ & $69,37 \pm 0,1$ & $61,98-78,7$ & $64,12 \pm 0,33$ & $56,21-69,14$ \\
\hline
\end{tabular}

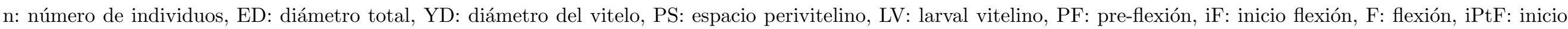

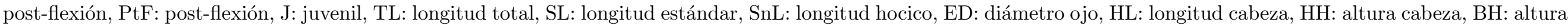

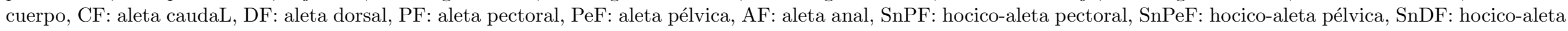
dorsal, SnAF: hocico-aleta anal, YL: longitud del vitelo, YH: altura del vitelo, AAU: aleta ausente, NV: no visible y S.D.: sin dato. 


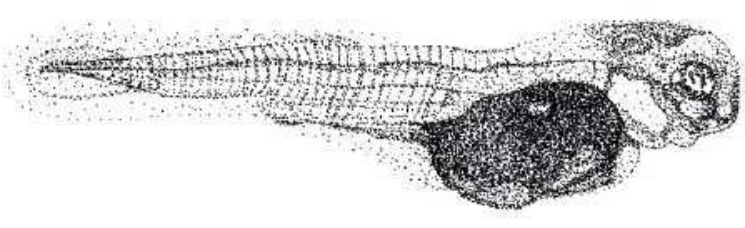

Figura 2A $\quad 500 \mathrm{um}$

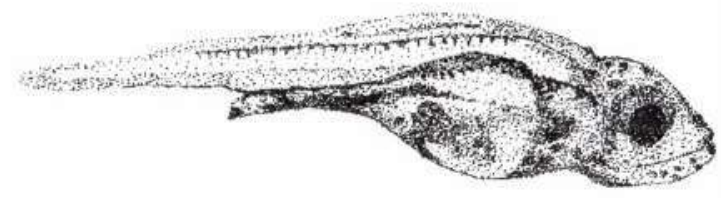

Figura 2C

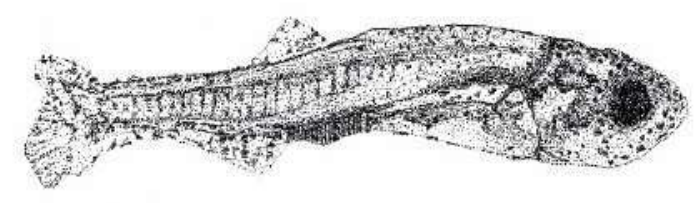

Figura 2E
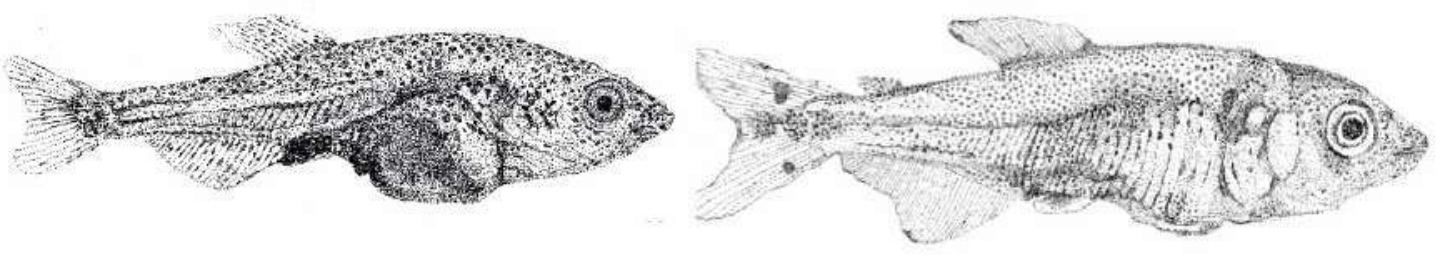

Figura $2 \mathrm{G}$

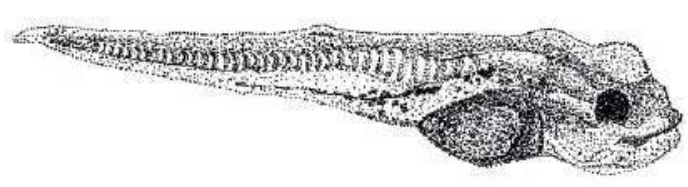

Figura 2B

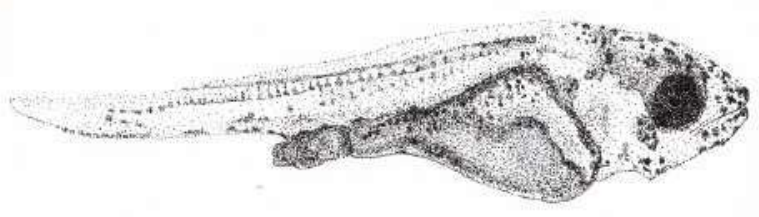

Figura 2D

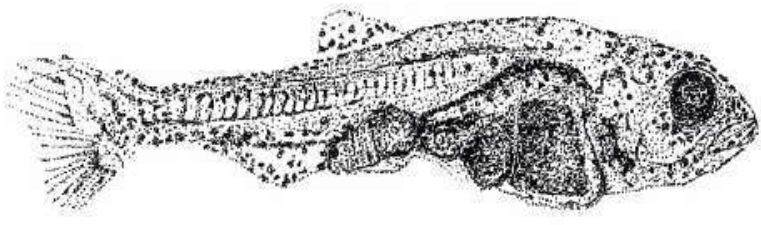

Figura $2 \mathrm{~F} \quad \underline{2 \mathrm{~mm}}$

Figura $2 \mathrm{~F}$

$2 \mathrm{~mm}$

Figura 2. Etapas de desarrollo larval de Brycon moorei. A. 0-HPE-LV; B. 24-HPE-LV; C. 25-53 HPE-PF; D. 56-71 HPE-iF; E. 82-110 HPE-F; F. 132-182 HPE-iPtF; G. 206-254 HPE-PtF; H. 278-326 HPE-J. 


\section{Estadio inicio de flexión (iF) (figura 2D-tabla 2)}

Periodo de tiempo comprendido entre las 56 hasta las 71HPE. Las larvas alcanzaron valores promedios en TL de $7,89 \mathrm{~mm}$, incremento que corresponde al $15 \%$ con relación a la etapa anterior; SL de 7,35 mm; ED de $0,46 \mathrm{~mm}$; BH de 1,45 mm, el cual incrementó en un $21,8 \%$ debido al consumo de alimento exógeno; HL de $1,32 \mathrm{~mm}$ y $\mathrm{HH}$ de $1,37 \mathrm{~mm}$. La relación ED/HL del $35,67 \pm 0,36 \%$, indica un ojo grande; HL/SL del $17,85 \pm 0,3 \%$ y BH/SL del 19,74 $\pm 0,6$, evidencian, respectivamente, una longitud de cabeza pequeña y una longitud del cuerpo largo. Se conservan las mismas características morfológicas de la etapa anterior, con ligera pigmentación puntiforme a nivel caudal.

\section{Estadio de flexión (F) (figura 2E-tabla 2)}

Periodo de tiempo comprendido entre las 82 hasta las 110 HPE. Las larvas alcanzaron valores promedios en TL de $9,85 \mathrm{~mm}$, incremento que corresponde al $24,8 \%$ con relación a la etapa anterior; SL de 9,26 $\mathrm{mm}$; ED de 0,66 mm; BH de 2,12 $\mathrm{mm}$, el cual aumentó en un $60,6 \%$ debido al consumo de alimento exógeno; HL de 1,96 mm y $\mathrm{HH}$ de 1,82 $\mathrm{mm}$. La relación $\mathrm{ED} / \mathrm{HL}$ del $34,2 \pm 0,24 \%$, indica un ojo grande; HL/SL del $21,14 \pm 0,36 \%$ y $\mathrm{BH} / \mathrm{SL}$ del $22,94 \pm 0,31$, indican respectivamente una longitud de cabeza y una longitud del cuerpo moderados. La notocorda alcanza la flexión total a las $95 \mathrm{HPE}$, siendo visible en el pedúnculo caudal con incremento notorio de la pigmentación dendrítica y puntiforme rodeando la notocorda y los radios de la aleta caudal primigenia; además, las aletas dorsal y anal comienzan su desarrollo y se observan también las aberturas nasales de forma alargada.

\section{Estadio de inicio de post-flexión (iPtF) (figura 2F- tabla 2)}

Periodo de tiempo comprendido entre las 123 hasta las 182 HPE. Las larvas alcanzaron valores promedios en TL de 13,81 mm, incremento que corresponde al 40,3\% con relación a la etapa anterior; SL de 12,44 mm; ED de 0,96 mm; BH de 3,13 mm, el cual incrementó en un $47,64 \%$, aunque menor comparado al incremento del $60,6 \%$ de la etapa de flexión, probablemente debido a la transición a la dieta seca; HL de $3,24 \mathrm{~mm}$, el cual incrementó un $66,8 \%$ con respecto a la etapa anterior y $\mathrm{HH}$ de $2,65 \mathrm{~mm}$. La relación $\mathrm{ED} / \mathrm{HL}$ del $30,55 \pm 0,21 \%$, indica un ojo moderado; HL/SL del 25,76 $\pm 0,38 \%$, y BH/SL del $24,91 \pm 0,4$, indican respectivamente una longitud de cabeza y una longitud del cuerpo moderados. La aleta primigenia ha sido absorbida en gran mayoría, solo se observa una tira delgada en el segmento preanal. Se observa un ensanchamiento en la base del pedúnculo caudal que formará la aleta adiposa. Se observan también pliegues y estrías a nivel del sistema digestivo y aletas anal, dorsal y caudal con radios. Los miómeros ya no son visibles a partir de esta etapa.

\section{Estadio de post-flexión (PtF) (figura 2G-tabla 2)}

Periodo de tiempo comprendido entre las 206 hasta las $254 \mathrm{HPE}$. Las larvas alcanzaron valores promedios en TL de 15,82 mm, incremento que corresponde al $14,55 \%$ con relación a la etapa anterior; SL de $13,68 \mathrm{~mm}$; ED de 1,1 mm; BH de 3,6 mm, el cual incremento en un $15,01 \%$ con respecto a la etapa anterior, menor probablemente debido a la transición a dieta seca; HL de $3,47 \mathrm{~mm}$ y $\mathrm{HH}$ de $3,29 \mathrm{~mm}$. La aleta caudal, tienen entre 24 a 26 radios; la aleta dorsal entre 9 a 10 radios, la aleta anal entre 20 a 22 radios; los radios de las aletas pectorales y pélvicas son difíciles de observar; con respecto a la pigmentación de las aletas, la única que no presentó patrones de pigmentación fue la aleta pectoral, independiente de su grado de desarrollo. La relación ED/HL del 29,91 $\pm 0,087 \%$, indica un ojo moderado; HL/SL del 27,28 $\pm 0,43 \%$, y BH/SL del 28,62 $\pm 0,57$, indican respectivamente una longitud de cabeza y una longitud del cuerpo moderados. Se mantienen la mayoría de las características morfológicas descritas en la etapa anterior.

\section{Estadio de juvenil (J) (figura 2H-tabla 2)}

Periodo de tiempo comprendido entre las 278 hasta las 326 HPE. Los juveniles alcanzaron valores promedios en TL de $25,66 \mathrm{~mm}$, incremento que corresponde al $62,2 \%$ con relación a la etapa anterior; SL de 21,49 mm; ED de 1,71 $\mathrm{mm}$; BH de 6,13 $\mathrm{mm}$, el cual incremento en un $70,2 \%$ con respecto a la etapa anterior, mayor probablemente debido a la adaptación a la dieta seca; HL de $6,33 \mathrm{~mm}$ y $\mathrm{HH}$ de $4,89 \mathrm{~mm}$. La aleta caudal, tienen entre 25 
a 27 radios; la aleta dorsal entre 11 a 13 radios, la aleta pectoral entre 15 a 16 radios, la aleta anal entre 29 a 32 radios y la pélvica entre 9 a 10. La relación $\mathrm{ED} / \mathrm{HL}$ del $27,17 \pm 0,071 \%$, indica un ojo moderado; HL/SL del 29,45 $\pm 0,095 \%$, y BH/SL del $28,56 \pm 0,082$, indican respectivamente una longitud de cabeza y una longitud del cuerpo moderados. Se observan escamas y todas las estructuras natatorias bien desarrolladas, incluyendo la aleta adiposa. Se observa además el punto negro característico en la base del pedúnculo caudal, con proyecciones rojizas sobre la aleta caudal e iridiscencias en todo el cuerpo, típicas en esta especie.

\section{Relaciones corporales lineales}

Los modelos de crecimiento de las diferentes variables corporales relacionadas para $B$. moorei, se presentan desde la figura 3 a la 8 ; en ellas se pueden observar las relaciones lineales entre longitud total (LT) y longitud estándar (LE) vs horas post-eclosión (HPE); LT vs LE; LE vs longitud de la cabeza $(\mathrm{LCBz})$;
LE vs altura de la cabeza $(\mathrm{HCBz})$; LE vs altura del cuerpo (H-Cuerpo) y LCBz vs diámetro del ojo (DIAM.Ojo). Además, se incluyen los intervalos de confianza (95\%), que determinan las relaciones de isometría o alometría positiva o negativa y si existen diferencias entre los rangos para todas las etapas de desarrollo temprano que incluye desde larva hasta juvenil. La figura 3 muestra la curva de crecimiento en LT y LE, con respecto al tiempo desde larva vitelina hasta juvenil, explicado a través del modelo logarítmico, con un coeficiente de determinación del $97 \%$ para LT y del $98 \%$ para LE, con una alta correlación entre las variables (98\%). Se indica también un periodo en el que el crecimiento fue menor (144 a $250 \mathrm{HPE}$ ), el cual coincide con el inicio del protocolo de alimentación con dieta seca (* $144 \mathrm{HPE})$.

La figura 4 indica la relación entre la longitud total y la longitud estándar, con una correlación superior al $90 \%$ y un coeficiente de determinación elevado en todas las etapas; existe isometría $(b=1)$ en $L V$, iF y F, es decir que LT y LE crecen en la misma

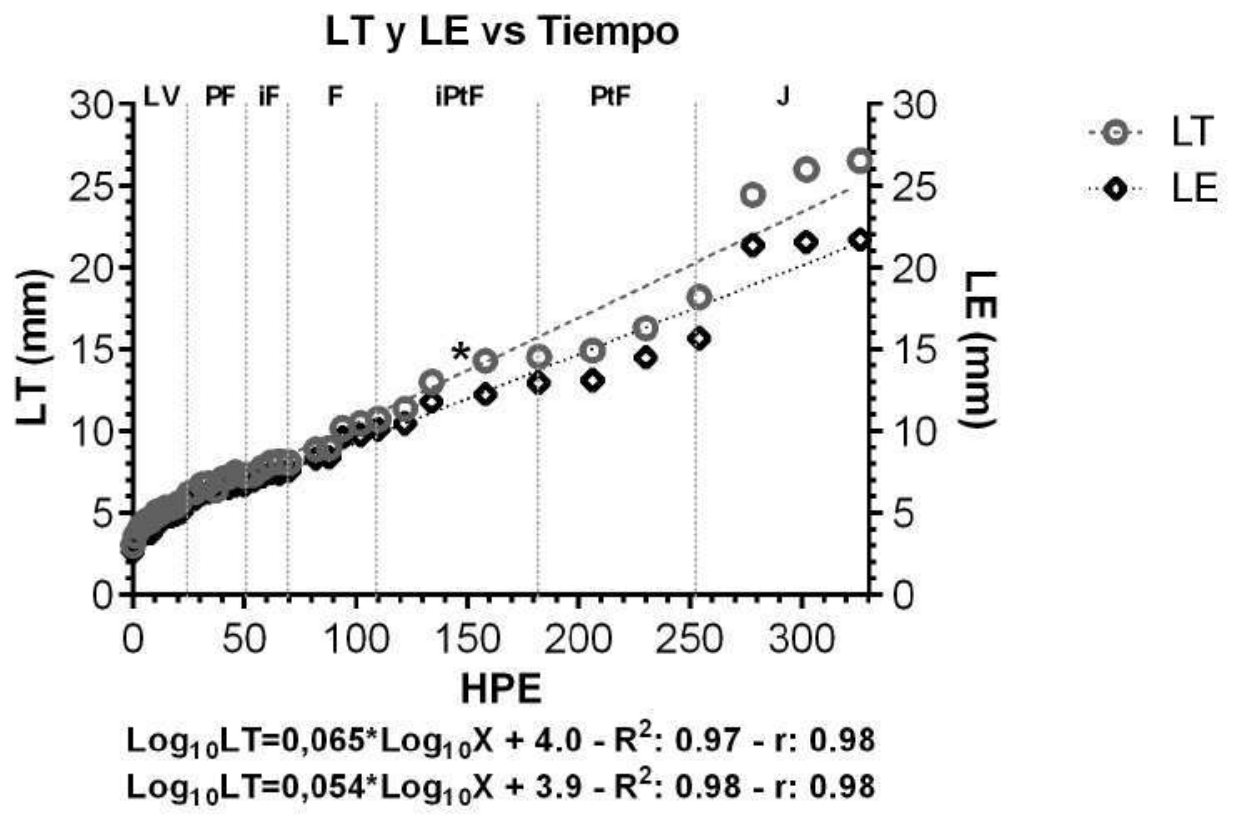

Figura 3. Relación lineal en logaritmo para LT y LE, con respecto a las HPE, durante el ciclo de desarrollo larval a juvenil en $B$. moorei $(n=470)$. Larval Vitelino (LV), Pre-Flexión (PF), Inicio Flexión (iF), Flexión $(F)$, Inicio de Post-Flexión (iPtF), Post-Flexión (PtF) y Juvenil $(\mathrm{J}) .(b<1) ;(b>1)$ y $(b=1)$ : alometría negativa, positiva o isometría. R2: Coeficiente de determinación. * indica el inicio de la dieta seca. 
proporción; para $\mathrm{PF}, \mathrm{iPtF}, \mathrm{PtF}$ y J la alometría es menor que 1, por lo tanto la LE no crece en la misma proporción que la LT; los intervalos de confianza permitieron establecer que la pendiente $(\boldsymbol{b})$ de $\mathrm{LV}$ tiene diferencias significativas con $\mathrm{PF}, \mathrm{iPtF}$ y J, a su vez la $\boldsymbol{b}$ de $\mathrm{PF}$ es diferente significativamente de $\mathrm{F}$ y $\boldsymbol{b}$ de $\mathrm{F}$ es significativamente diferente de $\mathrm{J}$.

La figura 5 indica la relación entre $\mathrm{LE}$ vs $\mathrm{LCBz}$, en la cual para las etapas LV, PF, iF e iPtF, la longitud de la cabeza crece de manera más rápida $(b>1)$ que la longitud estándar, a su vez los coeficientes de correlación y determinación son elevados, mostrando alta correlación entre las variables y un modelo confiable. Para F y PtF existe isometría $(b=1)$ entre las variables, con elevada correlación y un modelo ajustado; para J, aunque el crecimiento fue alométrico negativo, no se encontró relación entre las variables $(\mathrm{r}=24 \%)$ y el modelo no logra explicar dicha relación $\left(\mathrm{R}^{2}=6 \%\right)$ por la elevada dispersión de los datos. Los intervalos de confianza permitieron establecer que $\boldsymbol{b}$ de LV tiene diferencias significativas con F; PtF y J y que $\boldsymbol{b}$ de $\mathrm{J}$ tiene diferencias estadísticas significativas con todas las pendientes de las otras etapas.

La figura 6 muestra la relación entre la longitud estándar y la altura de la cabeza, donde LV e IPtF, presentan un crecimiento alométrico positivo $(b>1)$, con un modelo confiable y una alta correlación entre las variables; para $\mathrm{PF}, \mathrm{iF}$ y $\mathrm{F}$, existe isometría, con un $\mathrm{R}^{2}$ y $\mathrm{r}$ elevados a excepción de $\mathrm{PF}$, donde existió una mayor dispersión de los datos. Para PtF, el modelo no se ajustó por la elevada dispersión de los datos y en J, existe alometría negativa, con elevada correlación y confiabilidad del modelo.

La figura 7, representa la relación entre LE y la altura del cuerpo, la cual en la primera etapa (LV) muestra de manera clara el efecto del gasto de las reservas endógenas (vitelo), por lo cual la correlación es negativa, es decir entre la larva más crece más delgada se vuelve; luego se presenta isometría para $\mathrm{F}$, con elevada correlación; para PF y J también se presenta isometría, solo que la dispersión de los datos es alta, en este caso la correlación solo llega al $56 \%$; iF e iPtF, con elevada correlación y modelo ajustado la alometría es positiva.
Por último, la figura 8 , indica la relación entre la longitud de la cabeza y el diámetro del ojo, donde $\mathrm{LV}, \mathrm{iF}, \mathrm{iPtF}, \mathrm{PtF}$ y J, presentan elevada correlación y un modelo ajustado, mostrando alometría negativa; F, también presentó alometría negativa pero el modelo no es confiable, además presenta elevada dispersión de los datos, por lo tanto, la correlación entre las variables es baja; se presentó isometría en $\mathrm{PF}$, pero con baja correlación y elevada dispersión de los datos. Los intervalos de confianza permitieron establecer que $\boldsymbol{b}$ de LV tiene diferencias significativas con todas las etapas, en las relaciones indicadas por las figuras 5, 6 y 7; de manera general los rangos de los intervalos de confianza que más diferencias estadísticas tuvieron para todas las relaciones fueron los de la etapa juvenil.

Los valores de TCI para LT examinados en el transcurso del desarrollo de $B$. moorei, son mostrados en la figura 9 .

En las primeras $77 \mathrm{HPE}$, se observaron las mayores variaciones en la TCI para B. moorei, incluyendo los valores más altos entre la eclosión hasta la $3 \mathrm{HPE}$, con una caída hasta las 12 HPE y luego con una serie de altos y bajos; el valor más bajo se encontró a las $63 \mathrm{HPE}$, siguiendo con una ligera estabilidad, para luego, entre las 133 hasta las $181 \mathrm{HPE}$ tener otra caída.

\section{DISCUSIÓN}

Los cambios en el desarrollo temprano, son inherentes a cada especie (Balon, 1981) y a su vez, estos cambios están muy relacionados con las condiciones ambientales prevalentes. La temperatura, por ejemplo, puede acelerar o retrasar el desarrollo embrionario en los peces (Nakaghi, Neumann, Faustino, Mendes, y De Braga, 2014; Osse, y Van den Boogaart 1995). Para B. moorei la temperatura, entre otros, se mantuvo dentro de los rangos de confort para la especie (David-Ruales, y Castañeda-Álvarez, 2014). Muchas estructuras se convierten en claves para determinar parámetros de clasificación, como el tamaño del huevo (diámetro), el espacio perivitelino y el brote de la aleta pectoral, entre otras (Nakatani et al, 2001). B. moorei presenta un huevo pequeño $(2,72 \pm 0,12 \mathrm{~mm})$; el diámetro del huevo se puede 

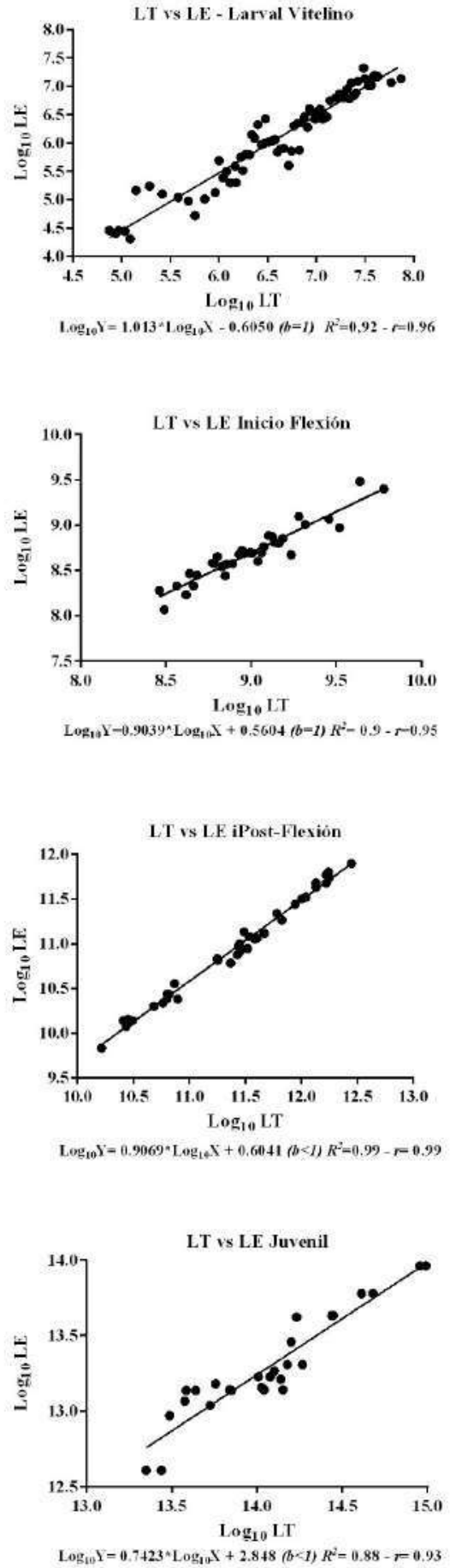

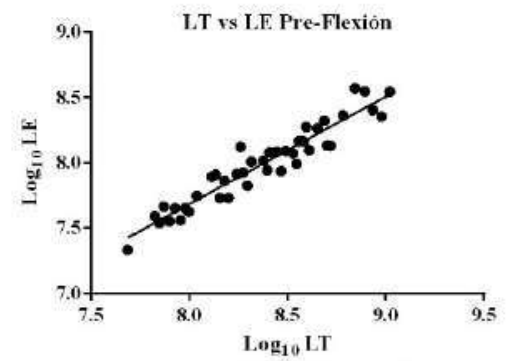

$\log _{10} \mathrm{Y}=0.8222^{\wedge} \log _{10} \mathrm{X}+1.105(b<1) R^{2}=0.91-r=0.95$
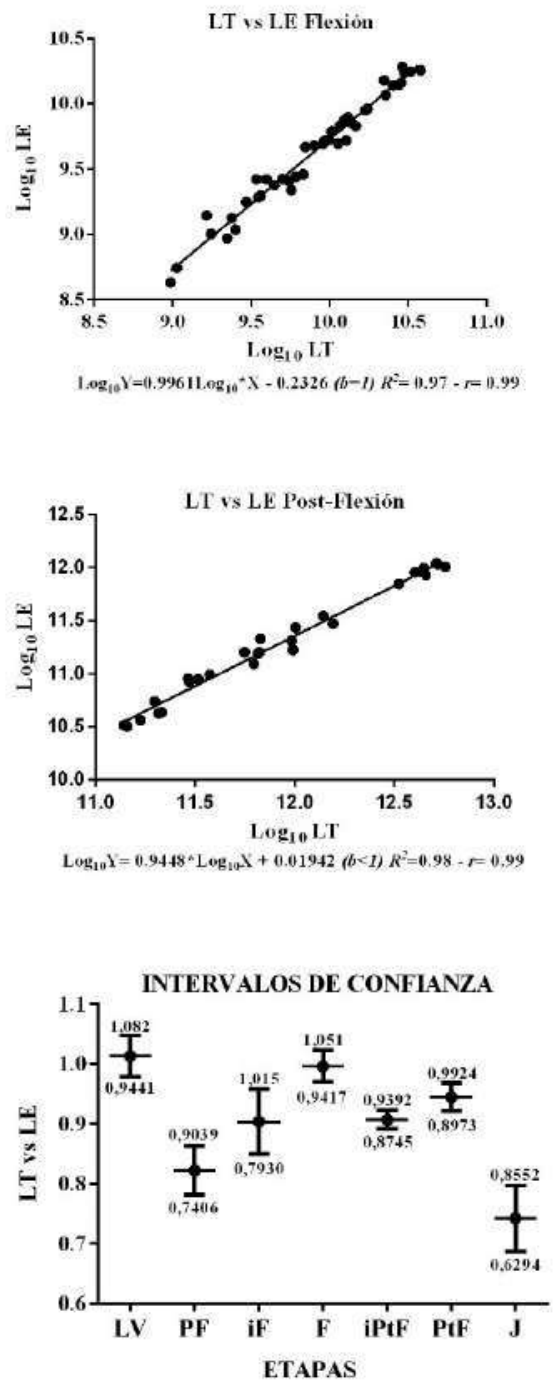

Figura 4. Relaciones lineares en logaritmo e intervalos de confianza (95\%) para LT y LE, durante las etapas Larval Vitelino $(n=180)$, Pre-Flexión $(n=90)$, inicio Flexión $(n=50)$, Flexión $(n=50)$, inicio Post-Flexión $(n=40)$, Post-Flexión $(n=30)$ y Juvenil $(n=30)$. Larva Vitelino (LV), Pre-Flexión (PF), Inicio Flexión (iF), Flexión $(F)$, Inicio de Post-Flexión (iPtF), Post-Flexión (PtF) y Juvenil $(\mathrm{J}) .(\mathrm{b}<1) ;(b>1)$ y $(b=1)$ : alometría negativa, positiva o isometría. $\mathrm{R}^{2}$ : Coeficiente de determinación y $\mathrm{r}$ : Coeficiente de correlación. 


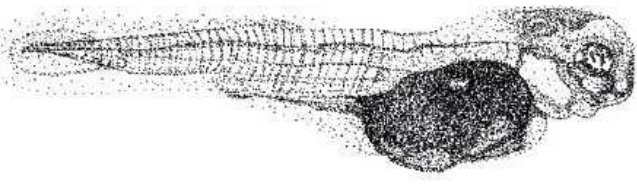

Figura 2A

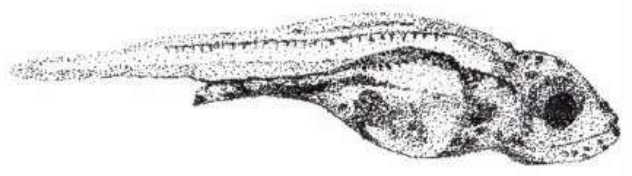

Figura 2C

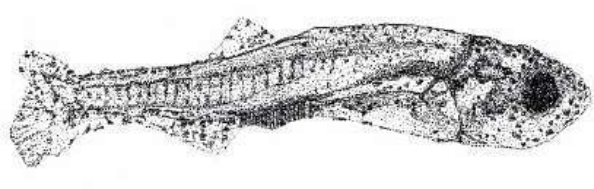

$2 \mathrm{~mm}$

Figura 2E

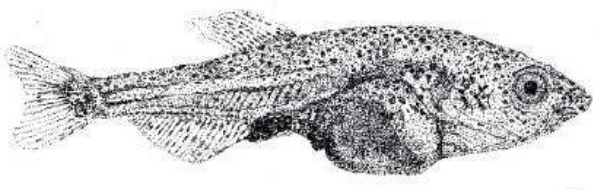

Figura 2G

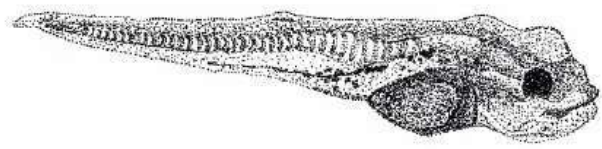

Figura 2B

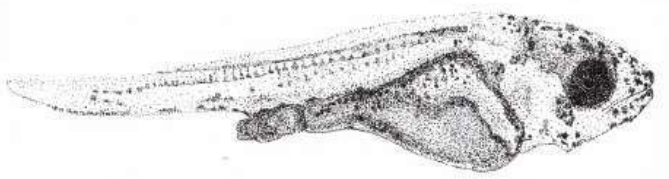

$2 \mathrm{~mm}$

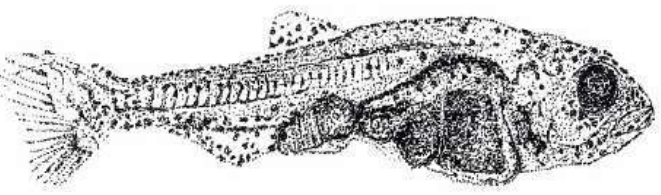

Figura $2 \mathrm{~F}$

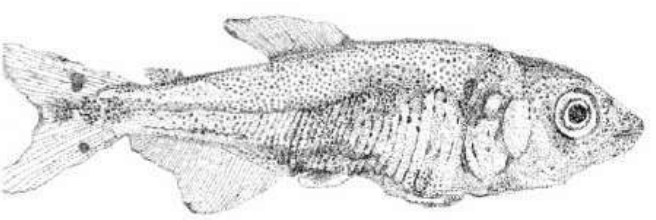

Figura 2H

Figura 5. Relaciones lineares en logaritmo e intervalos de confianza (95\%) para LE y LCBz, durante las etapas Larval Vitelino $(n=180)$, Pre-Flexión $(n=90)$, inicio Flexión $(n=50)$, Flexión $(n=50)$, inicio Post-Flexión $(n=40)$, Post-Flexión $(n=30)$ y Juvenil $(n=30)$. Larva Vitelino (LV), Pre-Flexión (PF), Inicio Flexión (iF), Flexión $(F)$, Inicio de Post-Flexión (iPtF), Post-Flexión (PtF) y Juvenil $(\mathrm{J}) .(\mathrm{b}<1) ;(b>1)$ y $(b=1)$ : alometría negativa, positiva o isometría. $\mathrm{R}^{2}$ : Coeficiente de determinación y $\mathrm{r}$ : Coeficiente de correlación. 


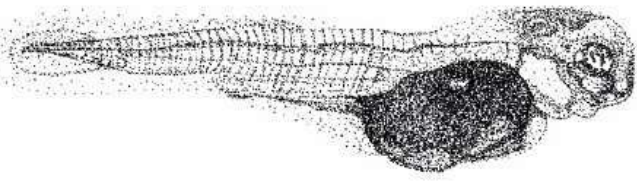

Figura 2A $\quad 500 \mathrm{um}$

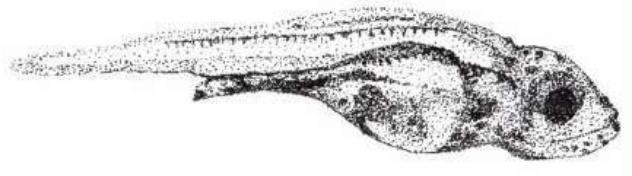

Figura 2C

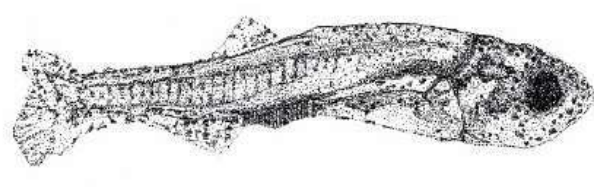

$2 \mathrm{~mm}$

Figura 2E
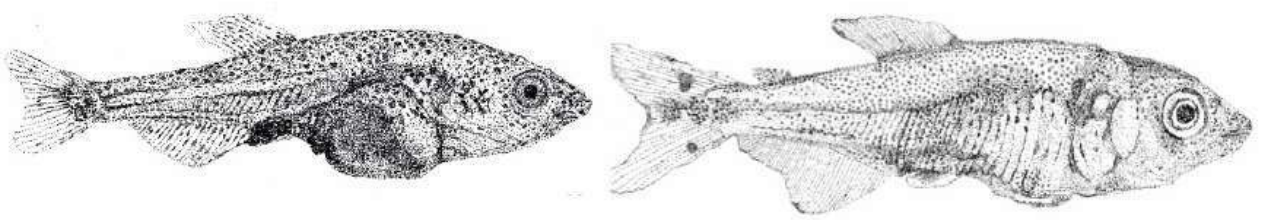

Figura $2 \mathrm{G}$

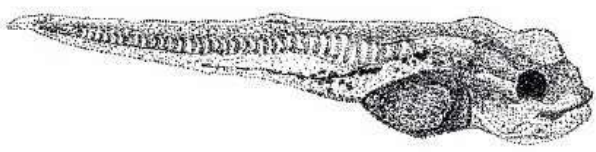

Figura 2B

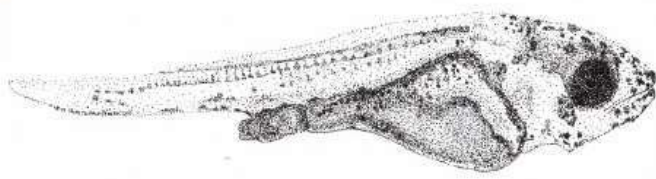

Figura 2D

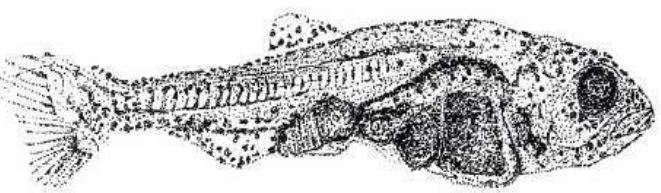

Figura $2 \mathrm{~F}$

\section{$2 \mathrm{~mm}$}

$2 \mathrm{~mm}$
Figura 2H

Figura 6. Relaciones lineares en logaritmo e intervalos de confianza (95\%) para LE y HCBz, durante las etapas Larval Vitelino $(n=180)$, Pre-Flexión $(n=90)$, inicio Flexión $(n=50)$, Flexión $(n=50)$, inicio Post-Flexión $(n=40)$, Post-Flexión $(n=30)$ y Juvenil $(n=30)$. Larva Vitelino (LV), Pre-Flexión (PF), Inicio Flexión (iF), Flexión $(F)$, Inicio de Post-Flexión (iPtF), Post-Flexión (PtF) y Juvenil $(\mathrm{J}) .(\mathrm{b}<1) ;(b>1)$ y $(b=1)$ : alometría negativa, positiva o isometría. $\mathrm{R}^{2}$ : Coeficiente de determinación y $\mathrm{r}$ : Coeficiente de correlación. 


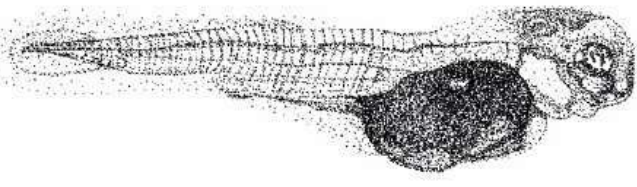

Figura 2A

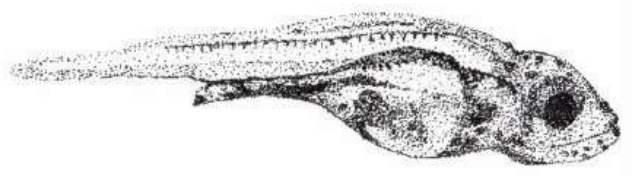

Figura 2C

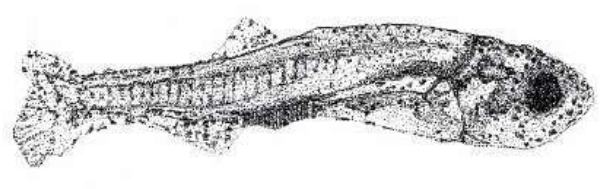

$2 \mathrm{~mm}$

Figura 2E

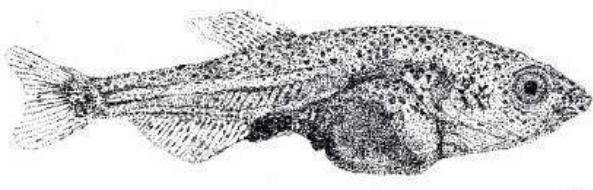

Figura 2G

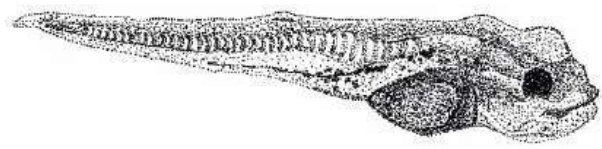

Figura 2B $\quad \underline{2 \mathrm{~mm}}$

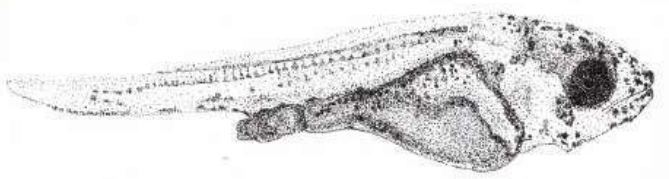

Figura 2D

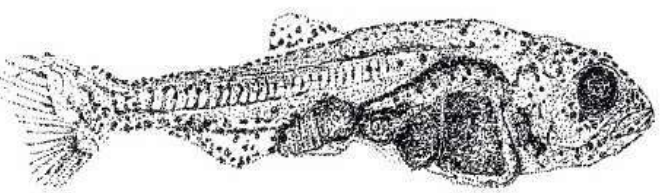

$2 \mathrm{~mm}$

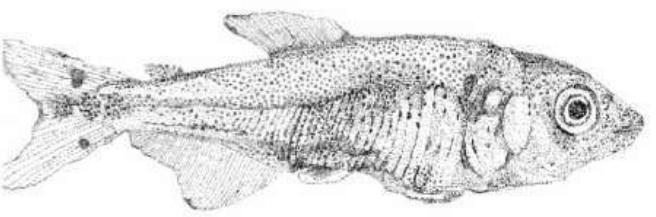

Figura 2H

Figura 7. Relaciones lineares en logaritmo e intervalos de confianza (95\%) para LE y H-Cuerpo, durante las etapas Larval Vitelino $(n=180)$, Pre-Flexión $(n=90)$, inicio Flexión $(n=50)$, Flexión $(n=50)$, inicio Post-Flexión $(n=40)$, Post-Flexión $(n=30)$ y Juvenil $(n=30)$. Larva Vitelino $(L V)$, Pre-Flexión (PF), Inicio Flexión (iF), Flexión $(F)$, Inicio de Post-Flexión (iPtF), Post-Flexión (PtF) y Juvenil $(\mathrm{J}) .(\mathrm{b}<1) ;(b>1)$ y $(b=1)$ : alometría negativa, positiva o isometría. $\mathrm{R}^{2}$ : Coeficiente de determinación y $\mathrm{r}$ : Coeficiente de correlación. 


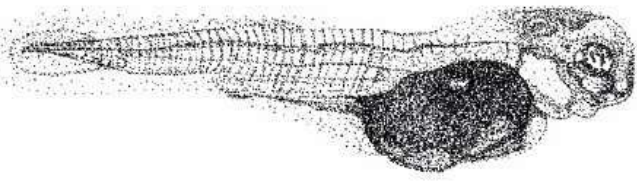

Figura 2A $\quad 500 \mathrm{um}$

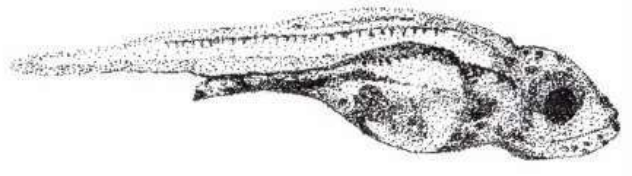

Figura 2C

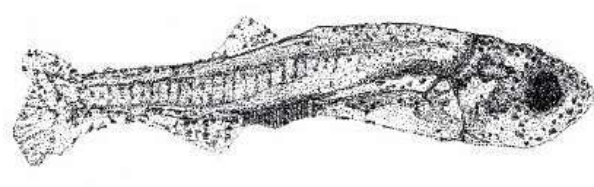

$2 \mathrm{~mm}$

Figura 2E

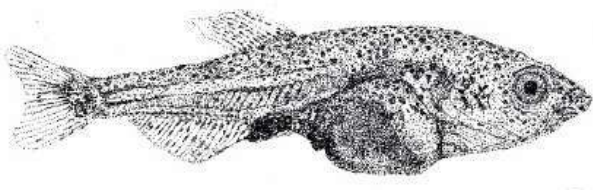

Figura $2 \mathrm{G}$

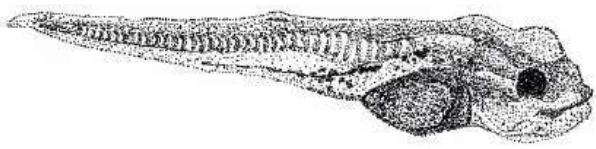

Figura 2B

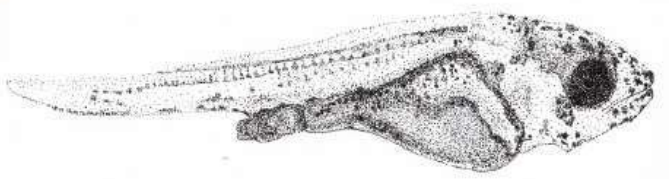

Figura 2D

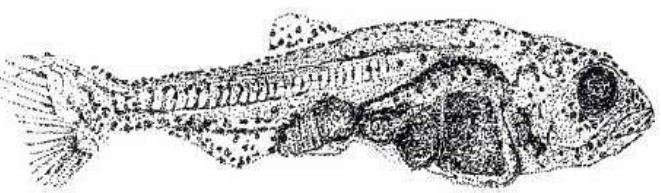

$2 \mathrm{~mm}$

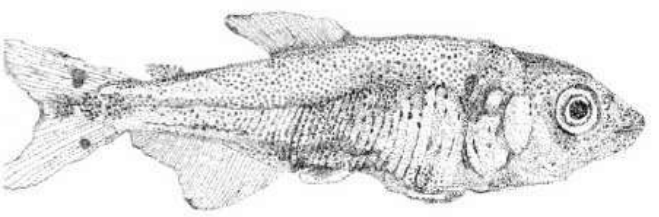

Figura $2 \mathrm{H}$

Figura 8. Relaciones lineares en logaritmo e intervalos de confianza (95\%) para LCBz y Diam. OJO, durante las etapas Larval Vitelino $(n=180)$, Pre-Flexión $(n=90)$, inicio Flexión $(n=50)$, Flexión $(n=50)$, inicio PostFlexión $(n=40)$, Post-Flexión $(n=30)$ y Juvenil $(n=30)$. Larva Vitelino $(L V)$, Pre-Flexión (PF), Inicio Flexión $(\mathrm{iF})$, Flexión (F), Inicio de Post-Flexión (iPtF), Post-Flexión (PtF) y Juvenil $(\mathrm{J}) .(\mathrm{b}<1)$; $(\mathrm{b}>1)$ y $(\mathrm{b}=1)$ : alometría negativa, positiva o isometría. $\mathrm{R}^{2}$ : Coeficiente de determinación y $\mathrm{r}$ : Coeficiente de correlación. 
relacionar con la estrategia reproductiva, por lo general especies que no presentan cuidado parental presentan este tipo de huevo (Kolm, y Ahnesjö, 2005), otros autores afirman que muchos de los peces teleósteos tienen esta característica, donde las reservas de vitelo se consumen rápidamente y las larvas producto de estas estructuras son capaces de alimentarse de presas pequeñas y adaptarse al medio que los rodea (Osse, 1990; Osse et al, 1997).

Dentro del género Brycon al eclosionar, ciertas características anatómicas se comparten, por ejemplo, ausencia de pigmentación, ano, intestino y vejiga gaseosa, además, la vesícula óptica está indiferenciada y la aleta embrionaria es hialina y rodea todo el cuerpo (Faustino, Makino, Neumann, y Nakaghi, 2018; Marques et al, 2017). Estos rasgos se observaron en el presente estudio y también en larvas de: $B$. insignis (piabanha) (Andrade-Talmelli, Kavamoto, Romagosa, y Fenerich-Verani, 2001). B. orbignyanus (piracanjuba) (Bettinelli-Nogueira, Lima-Godinho, y Pereira-Godinho, 2014; Raposo-Maciel et al, 2010; Reynalte-Tataje, Zaniboni-Filho, y Esquivel, 2004); B. amazonicus (yamú) (Da Silva et al, 2017; MiraLópez, Medina-Robles, Velasco-Santamaría, y CruzCasallas, 2007; Neumann, Paes, Mendes, Braga, y Nakaghi, 2018); B. cephalus (sinonimia=B. amazonicus) (matrinxã) (Romagosa, Narahara, y FenerichVerani, 2001; Sversut de Alexandre et al, 2010); $B$. gouldingi (matrinchã) (Faustino, Nakaghi, y Neu- mann, 2011; Faustino, Makino, Neumann, y Nakaghi, 2015) y B. nattereri (peripetinga) (Maria, NinhausSilveira, Orfão, y Viveiros, 2017). A diferencia de otros bricónidos (Andrade-Talmelli et al, 2001; MiraLópez et al, 2007; Nakatani et al, 2001; ReynalteTataje et al, 2004), la pigmentación del ojo en $B$. moorei inició más rápido (11 h después de la fertilización), no después de la eclosión. Se conoce que la visión es fundamental en la percepción de la distancia para la obtención de alimento y evitar la depredación (Hubbs, y Blaxter, 1986), además el género Brycon se caracteriza por tener ojos grandes y pigmentados, que indican mayor facilidad para la caza y evitar la depredación (Ceccarelli, 1997). En B. moorei, los patrones de pigmentación corporal, incrementan con el cambio de fase de crecimiento, intensificándose desde el inicio de la flexión, las máculas típicas se presentan a partir de la etapa de post-flexión localizadas en el pedúnculo caudal y en la parte posterior del opérculo; similares características son descritas para B. amazonicus (Gomes, y Urbinati, 2005).

En teleósteos existen patrones secuenciales de crecimiento (alometría) (Osse, y van den Boogaart, 1995), estos patrones se presentaron en B. moorei con diferencias dentro de cada etapa de desarrollo. Tales diferencias pueden estar relacionadas con la competencia por el alimento (Osse et al, 1997; van Snik, Van den Boogaart, y Osse, 1997), por el canibalismo exacerbado que presenta esta especie (Baras, 2005;

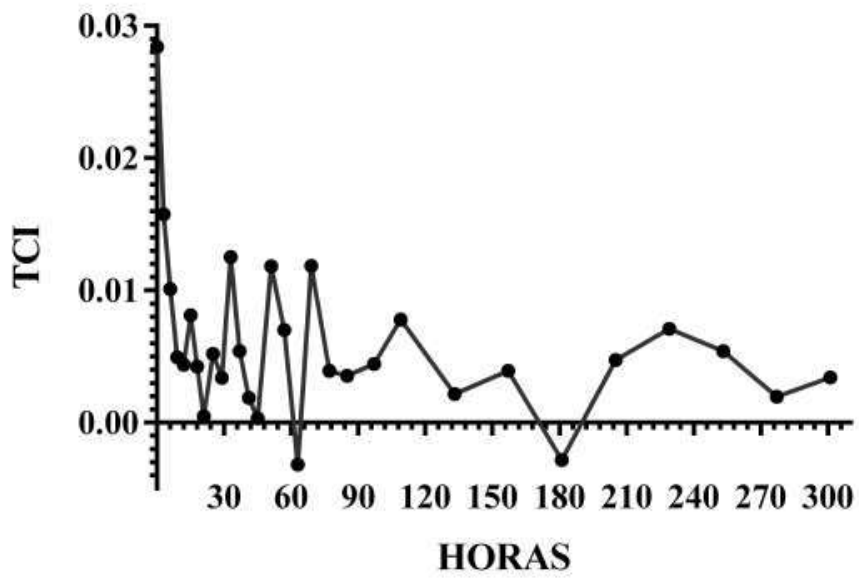

Figura 9. Tasa de crecimiento instantáneo (TCI) para LT durante el desarrollo larval de Brycon moorei. 
Baras, y Jobling, 2002; Baras, y Lucas, 2010; Baras, Maxi, Ndao, y Mélard, 2000a; Baras et al, 2000b;) y por factores medioambientales (Fuiman, y Higgs, 1997; Fuiman, Poling, y Higgs, 1998).

El crecimiento larval por lo general sigue el modelo exponencial (Fuiman, 1983). En el presente estudio, los patrones de crecimiento alométrico fueron explicados a través de la transformación de este modelo, el valor de LT para $B$. moorei al final del periodo experimental fue de 25,66 $\pm 0,64 \mathrm{~mm}$; usando el mismo modelo, se reportó un valor similar en $B$. orbignyanus $(24,9 \mathrm{~mm})$ (Bettinelli-Nogueira et al, 2014); para la etapa de post-flexión en el presente estudio se encontró un valor de 15,82 $\pm 0,41 \mathrm{~mm}$, se reportan valores similares en la misma etapa de desarrollo para larvas de $B$. insignis con 16,48 $\pm 1,38$ $\mathrm{mm}$, larvas de Salminus brasiliensis $(14,84 \pm 1,70$ $\mathrm{mm}$ ) (Souza et al, 2017) y P. vimboides con 15,68 \pm $1,0 \mathrm{~mm}$ (Souza et al, 2016) y, sin reportar el modelo, menor valor para $B$. amazonicus, con una LT de 18,89 $\pm 3,75$ (Neumann et al, 2018); menor valor para $B$. orthotaenia al día 7 con 9,58 $\pm 0,67 \mathrm{~mm}$ (ZeferinoGomes, Sato, Rizzo, y Bazzoli, 2011), siendo que en B. moorei en el mismo periodo alcanzaba los 13,66 \pm $1,27 \mathrm{~mm}$.

Con respecto al crecimiento en el periodo experimental para $B$. moorei reflejado en la relación de la LT y LE versus HPE, se observa entre las 150 y las 250 HPE una queda en el crecimiento, comparado con las etapas anteriores y la posterior a las $250 \mathrm{HPE}$, este patrón en el crecimiento puede estar influenciado por el cambio en el protocolo de alimentación, el cual coincide con el inicio de la dieta; se conoce que el crecimiento y la composición química corporal de los peces pueden ser influenciados por la manipulación de los nutrientes de las dietas (Dabrowski, 1984), además en larvas de desarrollo indirecto la transición a dieta seca, después del alimento vivo, puede presentar este tipo de comportamientos, por lo que se recomienda el diseño de protocolos de alimentación (Portella, Leitão, Takata, y Lopes, 2012), asociado al seguimiento de la calidad del agua, el tipo de contendor y aspectos etológicos de la especie (DavidRuales, y Castañeda-Álvarez, 2010)

Respecto a la relación LT vs LE, en B. moorei, se encontraron variaciones entre isometría y alometría negativa con rangos muy parecidos, este resultado puede ser el reflejo del crecimiento de la aleta caudal; al respecto, Osse et al (1997) indican que el desarrollo de esta estructura está relacionado con las respuestas a la captura de presas y la huida de depredadores. La correspondencia entre la $\mathrm{LCBz}$ y $\mathrm{HCBz}$ vs $\mathrm{LE}$, tubo en principio alometría positiva, con acelerado crecimiento hasta $\mathrm{PtF}$, indicando priorización y diferenciación de estructuras bucales, sensoriales y otras que tienen que ver con el consumo de alimento y el cambio de respiración cutánea a branquial (funciones vitales) (Fuiman, 1983; 2002; Fuiman et al, 1998; Osse, 1990; Osse, y van den Boogaart, 1995; Osse et al, 1997; Van Snik et al, 1997; Vandewalle, Germeau, Besancenet, Parmentier, y Baras, 2005). Este tipo de desarrollo también se presentó en $B$. amazonicus (Neumann et al, 2018; Sampaio-Nakauth, Villacorta-Correa, Figueiredo, Bernardino, y França, 2016), B. cephalus (Romagosa et al, 2001), B. orbignyanus (Bettinelli-Nogueira et al, 2014) y en otras especies como Prochilodus vimboides (Souza et al, 2016), Pseudoplatystoma reticulatum (Andrade et al, 2016), Paralichthys californicus (Gisbert et al, 2002), Acipenser medirostris (Gisbert, y Doroshov, 2006), Ciprinus carpio, Clarias gariepinus (Van Snik et al, 1997) y para Nannacara anomala (Kupren, Prusi, Arski, Krejszeff, y Kucharczyk, 2014). En general, para la relación LCBz vs DIAM.OJO hubo una marcada alometría negativa, coincidiendo con la especie P. vimboides (Souza et al, 2016) y también para la especie $P$. reticulatum cuando comparado con la LT. $\mathrm{Al}$ respecto, se conoce que los cambios morfológicos se asocian con variaciones alométricas en órganos y otras partes del cuerpo con relación a las presiones medioambientales; sin embargo, órganos como el ojo y el cerebro crecen a tasas asociadas más con la edad que con el tamaño del cuerpo. Esto implica provisión de habilidades sensoriales y cognitivas necesarias para la sobrevivencia (Gonzalez-Voyer, Winberg, y Kolm, 2009; Pankhurst, y Montgomery, 1994). La interacción entre HCuerpo vs LE, pasó de alometría negativa en la etapa $L V$, alcanzando la isometría en la etapa de PF, para luego ser predominante la alometría positiva. El resultado en LV es directamente relacionado con el alto consumo de vitelo, el cual disminuye el tamaño del saco vitelino, influenciando directamente sobre la altura del cuerpo, resultados 
iguales se observaron en las especies: $P$. vimboides (Souza et al, 2016); C. carpio y C. gariepinus (Van Snik et al, 1997).

Con respecto a la TCI, el mayor valor al inicio de la eclosión se deba al hecho de presentar todas las reservas endógenas, con la correspondiente caída, dada al consumo de las mismas debido a la elevado metabolismo por la formación de estructuras relacionadas con la locomoción y la alimentación (Osse et al, 1997). Además del estrés generado por el ambiente, el protocolo de alimentación y hacia las $22 \mathrm{~h}$ posteclosión el incremento exacerbado del canibalismo, que se extiende durante todo el proceso. Las tasas de crecimiento no vuelven a su estado inicial debido al gasto energético por la actividad de caza y/o depredación. La otra caída que se observa hacia las $180 \mathrm{HPE}$, puede estar asociada probablemente con la transición alimenticia a dieta seca, en adelante se observa una ligera estabilidad hasta el final del experimento.

Los valores morfológicos reportados para $B$. moorei (tabla 2), son similares a otros especímenes de la familia Characidae y también del género Brycon por ejemplo: B. orbignyanus (Nakatani et al, 2001), S. brasiliensis (dorado), Leporinus steindachneri (piau), P. lineatus (curimba) y B. insignis (piabanha) (Souza et al, 2017), P. vimboides (curimbatá) (Souza et al, 2016) y mayores al ser comparados con las especies B. hilarii (piraputanga) (Oliveira et al, 2012), Pseudoplatystoma reticulatum (bagre) (Andrade et al, 2016) y Pimelodus britskii (bagre) (Almeida et al, 2018).

El modelo de crecimiento y las relaciones morfométricas encontradas para B. moorei, sugiere la priorización de estructuras que le dan a esta especie la habilidad para la locomoción y la captura de alimento en respuesta a ser considerada la especie de agua dulce con mayor canibalismo reportado para larvas de peces de agua dulce (Baras, y Jobling, 2002; Baras y Lucas, 2010; Baras et al, 2000b). Esta priorización es determinante para la sobrevivencia de los estadios iniciales en peces (Osse, 1990; Osse et al, 1997). Se conoce de la dificultad que se presenta para determinar el desarrollo temprano de las especies del neotrópico, la poca información, escasez de claves taxonómicas y el gran parecido entre especies en estos estados, constituye uno de los principales cuellos de botella a la hora de realizar este tipo de estudios (Lopes, Oliveira, Bialetzki, y Agostinho, 2015). Con los resultados obtenidos, se puede indicar que el manejo en confinamiento de la especie $B$. moorei, necesita de un protocolo de alimentación que incluya alimento vivo de diferentes tamaños y, que la transición a dieta seca, debe hacerse de manera paulatina, sin dejar en principio la co-alimentación con alimento vivo, de acuerdo con los patrones de crecimiento presentados; este procedimiento permite el mejor manejo para una especie de alto canibalismo, asegurando mejores tasa de sobrevivencia, que permiten certificar su adaptación para la acuicultura. Además, las relaciones morfométricas presentadas, se constituyen en valiosa información que servirá como claves merísticas para los estudios de ictioplancton, toda vez que la especie está declarada como vulnerable.

\section{AGRADECIMIENTOS}

Esta investigación contó con el apoyo del Centro de Investigaciones de la Universidad de los Llanos, dentro del proyecto de formación doctoral titulado: "Desarrollo temprano de la especie Brycon moorei - Steindachner, 1878 (dorada del Magdalena): aspectos embrionarios, morfométricos, histológicos e histoquímicos, parámetros productivos y actividad de las enzimas digestivas en función de la dieta".

\section{CONFLICTO DE INTERESES}

Los autores declaran no tener conflicto de intereses.

\section{REFERENCIAS}

Almeida, M.C., Message, H.J., Sanches, P.V., Baumgartner, D., Bombardelli, R.A., Baumgartner, G., y Langeani, F. (2018). Larval development of mandipintado Pimelodus britskii (Siluriformes: Pimelodidae). Journal of Fish Biology, 94, 320-324. DOI:10.1111/jfb.13884

Andrade-Talmelli, E.F., Kavamoto, E.T., Romagosa, E., y Fenerich-Verani, N. (2001). Embrionic and larval development of the Piabanha, Brycon insignis, Steindachner, 1876 (Pisces, Characidae). Boletim do Instituto de Pesca, 27, 21-28. DOI:10.1007/s00330-006-0500-2

Andrade, F.F., Lima, A.F., Assumpção, L., Makrakis, S., Kasai, R.I.D., y Makrakis, M.C. (2016). Characterization of 
the early development of Pseudoplatystoma reticulatum Eigenmann \& Eigenmann, 1889 (Siluriformes: Pimelodidae) from the Paraguay River Basin. Neotropical Ichthyology, 14, 2-15. DOI:10.1590/1982-0224-20150032

Balon, E.K. (1986). Types of feeding in the ontogeny of fishes and the life-history model. Environmental Biology of Fishes, 16, 11-24. DOI:10.1007/BF00005156

Balon, E.K. (1981). Saltatory processes and altricial to precocial forms in the ontogeny of fishes. American Zoologist, 21, 573-596. Retrieved from URL: http://www.jstor.org/ stable/3882651

Baras, E. (2005). Minimización del canibalismo en especies de peces con larvas piscívoras: estrategias y éxitos con el carácido Brycon moorei. In J.F, Renno, C. García, F, Duponchelle, J. Nuñez (Eds.). Biología de las poblaciones de peces de la amazonía y piscicultura (Cap 3, pp. 227-233). Iquitos, Perú: Red de Investigación sobre la Ictiofauna Amazónica.

Baras, E., y Jobling, M. (2002). Dynamics of intracohort cannibalism in cultured fish. Aquaculture Research, 33, 461479. DOI:10.1046/j.1365-2109.2002.00732.x

Baras, E., y Lucas, M.C. (2010). Individual growth trajectories of sibling Brycon moorei raised in isolation since egg stage, and their relationship with aggressive behaviour. Journal of Fish Biology, 77, 985-997. DOI:10.1111/j.1095-8649.2010.02740.x

Baras, E., Maxi, M.Y.J., Ndao, M., y Mélard, C. (2000a). Sibling cannibalism in dorada under experimental conditions. II. Effect of initial size heterogeneity, diet and light regime on early cannibalism. Journal of Fish Biology, 57, 1021-1036. DOI:10.1006/jfbi.2000.1365

Baras, E., Ndao, M., Maxi, M.Y.J., Jeandrain, D., Thomé, J.P., Vandewalle, P., y Mélard, C. (2000b). Sibling cannibalism in dorada under experimental conditions. I. Ontogeny, dynamics, bioenergetics of cannibalism and prey size selectivity. Journal of Fish Biology, 5\%, 1001-1020. DOI:10.1006/jfbi.2000.1366

Bettinelli-Nogueira, L., Lima-Godinho, A., y Pereira-Godinho, H. (2014). Early development and allometric growth in hatchery-reared characin Brycon orbignyanus. Aquaculture Research, 45, 1004-1011. DOI:10.1111/are.12041

Ceccarelli, P.S. (1997). Canibalismo em larvas de matrinxã Brycon cephalus (Gunther, 1869). (Tesis do Mestrado em Ciências Biológicas). Universidade Estadual Paulista - UNESP: Botucatu, Brasil.

Da Silva, C.R., Pereira Dos Santos, M., Senhorini, J.A., Paes, M.D.C.F., Valentin, F.N., Fujimoto, T., Do Nascimento, N.F., Yasui, G.S., y Nakaghi, L.S.O. (2017). The effect of temperature on the initial development of Brycon amazonicus Spix \& Agassiz, 1829 as tool for micromanipulation of embryos. Zygote, 25, 637-651. DOI:10.1017/S096719941700051X

Dabrowski, K. (1984). The feeding of fish larvae: present "state of the art" and perspectives. Reproduction Nutrition Développemen, 24, 807-833. DOI:10.1051/rnd:19840701

David-Ruales, C.A., y Castañeda-Álvarez, G.D. (2010). Larvicultura de peces comerciales en sistemas de recirculación, In: L.F, Garcés-Giraldo (Ed.). Perspectivas y avances de investigación (Cap 12, pp 199-215). Caldas, Ant, Colombia: Artes y Letras S.A.S. Corporación Universitaria
Lasallista.

David-Ruales, C.A., y Castañeda-Álvarez, G.D. (2014). Evaluation of a recirculation system for Dorada (Brycon moorei-Steindachner 1878) larvae in the Magdalena River. Revista CES Medicina Veterinaria y Zootecnia, 9, 179189. Retrieved from URL http://www.scielo.org.co/pdf/ cmvz/v9n2/v9n2a04.pdf

Devlin, R.H., Vandersteen, W.E., Uh, M,. y Stevens, E.D. (2012). Genetically modified growth affects allometry of eye and brain in salmonids. Canadian Journal of Zoology, 90, 193-202. DOI:10.1139/z11-126

Fuiman, L.A. (1983). Growth gradients in fish larvae. Journal of Fish Biology, 23, 117-123. DOI:10.1111/j.10958649.1983.tb02886.x

Faustino, F. (2010). Desenvolvimento embrionário e larval de Brycon gouldingi (Teleostei, Characidae). (Tesis do Mestre em Aquicultura). Universidad Estadual PaulistaUNESP: Botucatu, Brasil.

Faustino, F., Nakaghi, L.S.O., y Neumann, E. (2011). Brycon gouldingi (Teleostei, Characidae): aspects of the embryonic development in a new fish species with aquaculture potential. Zygote, 19, 351-363. DOI:10.1017/S0967199410000535

Faustino, F., Makino, L.C., Neumann, E., y Nakaghi, L.S.O. (2015). Morphological and morphometric aspects of early life stages of piabanha Brycon gouldingi (Characidae). Journal of Fish Biology, 86, 1491-1506. DOI:10.1111/jfb.12652

Faustino, F., Makino, L.C., Neumann, E., y Nakaghi, L.S.O. (2018). Ultrastructure aspects of Brycon gouldingi (Teleostei, Characidae) related to swimming ability and feeding during larval development. Journal of Fish Biology, 92, 1560-1573. DOI:10.1111/jfb.13612

Fuiman, L.A., y Higgs, D.M. (1997). Ontogeny, growth, and the recruitment process, In R.C, Chambers, E.A,Trippel (Eds.). Early life history and recruitment in fish populations (Cap 8, pp. 225-249). London: Chapman and Hall.

Fuiman, L.A., Poling, K.R., y Higgs, D.M. (1998). Quantifying developmental progress for comparative studies of larval fishes. Copeia, 3, 602-611. DOI:10.2307/1447790

Gilbert, S.F., y Bolker, J.A. (2003). Ecological developmental biology: preface to the symposium ${ }^{1}$. Evolution and Development, 5, 3-8. DOI:10.1046/j.1525-142X.2003.03002.x

Gisbert, E. (1999). Early development and allometric growth patterns in Siberian sturgeon and their ecological significance. Journal of Fish Biology, 54, 852-862. DOI:10.1111/j.1095-8649.1999.tb02037.x

Gisbert, E., y Doroshov, S.I. (2006). Allometric growth in green sturgeon larvae. Journal of Applied Ichthyology, 22, 202207. DOI:10.1111/j.1439-0426.2007.00952.x

Gisbert, E., Merino, G., Muguet, J.B., Bush, D., Piedrahita, R.H., and Conklin, D.E. (2002). Morphological development and allometric growth patterns in hatchery-reared California halibut larvae. Journal of Fish Biology, 61, 1217-1229. DOI:10.1111/j.1095-8649.2002.tb02466.x

Gomes, L.C., y Urbinati, E.C. (2005). Matrinxã (Brycon amazonicus), In B, Baldisseroto, L.C, Gomes (Eds.). Espécies nativas para piscicultura no brasil. (Cap 7, pp. 149-174). Santa Maria, Brasil: Editorial da UFSM

Gonzalez-Voyer, A., Winberg, S., y Kolm, N. (2009). Brain 
structure evolution in a basal vertebrate clade: evidence from phylogenetic comparative analysis of cichlid fishes. BMC Evolutionary Biology, 9, 238. DOI:10.1186/14712148-9-238

Hubbs, C., y Blaxter, J.H.S. (1986). Ninth Larval Fish Conference. Transactions of the American Fisheries Society, 115, 98-114. DOI:10.1577/15488659(1986) $115<98:$ NLFCDO $>2.0 . \mathrm{CO} ; 2$

Kendall, Jr., A.W., Ahlstrom, E.H., y Moser, H.G. (1983). Early life history stages of fishes and their characters, In H.G, Moser, W. J, Richards, D.M, Cohen, M.P, Fahay, A.W, Kendall, Jr., S.L, Richardson (Eds.). Ontogeny and systematic of fishes (Cap 1, pp. 15-20). La Jolla, Califrornia. USA: American Society of Ichthyologists and Herpetologists.

Kolm, N., y Ahnesjö, I. (2005). Do egg size and parental care coevolve in fishes? Journal of Fish Biology, 66, 1499-1515. DOI:10.1111/j.0022-1112.2005.00777.x

Kimmel, C.B., Ballard, W.W., Kimmel, S.R., Ullmann, B., y Schilling, T.F. (1995). Stages of embryonic development of the zebrafish. Developmental Dynamics, 203, 253-310. DOI:10.1002/aja.1002030302

Kupren, K., Prusi, M., Arski, D., Krejszeff, S., y Kucharczyk, D. (2014). Early development and allometric growth in Nannacara anomala Regan, 1905 (Perciformes: Cichlidae) under laboratory conditions. Neotropical Ichthyology, 12, 659-665. DOI:10.1590/1982-0224-20130104

Leiby, M.M. (1984). Life history and ecology of pelagic fish eggs and larvae. In K.A. Steidinger and L.M. Walker (Eds.). Marine plankton life cycle strategies (Cap 1, pp. 121-140). Boca Raton, FL: CRC Press.

Lopes, T.M., Oliveira, F.G., Bialetzki, A., y Agostinho, A.A. (2015). Early development in the mouth-brooding cichlid fish Satanoperca pappaterra (Perciformes: Cichlidae). Revista de Biología Tropical, 63, 139-153. Retrieved from URL https://www.scielo.sa.cr/scielo.php?pid= S0034-77442015000100011\&script=sci_arttext\&tlng=en

Mansano, C.F., De Stéfani, M., Pereira, M., Nascimento, T., y Macente, B. (2014). Morphometric growth characteristics and body composition of bullfrog tadpoles in captivity. Semina Ciências Agrárias, 35, 2817-2830. DOI:10.5433/1679-0359.2014v35n5p2817

Mansano, C.F., Macente, B.I., Ullah-Khan, K., do Nascimento, T.M., da Silva, E.P., Kazue-Sakomura, N. y Ferdes, J.B. (2017). Morphometric growth characteristics and body composition of fish and amphibians, In: P.M, ParesCasanova (Ed.). New Insights into Morphometry Studies (Cap 1, pp. 7-28). London, Inglaterra: Intech Open.

Maria, A.N., Ninhaus-Silveira, A., Orfão, L.H., y Viveiros, A.T.M. (2017). Embryonic development and larval growth of Brycon nattereri Günther, 1864 (Characidae) and its implications for captive rearing. Zygote, 25, 711718. DOI:10.1017/S0967199417000594

Marques, C., Faustino, F., Bertolucci, B., Do Carmo Faria Paes, M., Valentin, F.N., y Nakaghi, L.S.O. (2017). Structural and ultrastructural description of larval development in Zungaro jahu. Zygote, 25, 149-159. DOI:10.1017/S0967199416000423

Mira-López, T.M., Medina-Robles, V.M., Velasco-Santamaría, Y.M., y Cruz-Casallas, P.E. (2007). Valores morfomé- tricos en larvas de yamú Brycon amazonicus (Pisces: Characidae) obtenidas con semen fresco y crioconservado. Actualidades Biológicas, 29, 209-219. Retrieved from URL https://revistas.udea.edu.co/index.php/ actbio/article/view/329340

Mojica, J.I., Usma, J.S., Álvarez-León, R., y Lasso C.A (Eds). (2012). Libro rojo de peces dulceacuícolas de Colombia. (pp. 319). Bogotá, Colombia: Instituto de Investigación de Recursos Biológicos Alexander von Humboldt, Instituto de Ciencias Naturales de la Universidad Nacional de Colombia, WWF Colombia y Universidad de Manizales.

Nakatani, K., Agostinho, A.A., Baumgartner, G., Bialetzki, A., Sanches P.V., y Cavicchioli, M. (Eds). (2001). Ordem Characiformes. Ovos e larvas de peixes de agua doce: desenvolvimento e manual de identificação. (Cap 9, pp. 120-124). Maringa, PR. Brasil: Editorial EDUEM.

Nakaghi, L.S.O., Neumann, E., Faustino, F., Mendes, J.M.R., y de Braga, F.M. (2014). Moments of induced spawning and embryonic development of Brycon amazonicus (Teleostei, Characidae). Zygote, 22, 549-557. DOI:10.1017/S0967199413000130

Neumann, E. (2008). Desenvolvimento inicial de Jatuarana, Brycon amazonicus (Teleostei, Characidae). (Tese de Doutorado em Aqüicultura). Universidade Estadual Paulista. Centro de Aqüicultura da UNESP. Brasil.

Neumann, E., Paes, M.C.F., Mendes, J.M.R., Braga, F.M.S., y Nakaghi, L.S.O. (2018). Larval development of Brycon amazonicus (Teleostei, Bryconidae) with a focus on locomotory, respiratory and feeding structures. Journal of Fish Biology, 93, 1141-1150. DOI:10.1111/jfb.13832

Oliveira, F.G. de, Bialetzki, A., Gomes, L.C., Santin, M., y Taguti, T.L. (2012). Desenvolvimento larval de Brycon hilarii (Characiformes, Characidae). Iheringia. Série Zoologia, 102, 62-70. DOI:10.1590/S0073-47212012000100009

Orton, G.L. (1953). The systematics of vertebrate larvae. Systematic Zoology, 2, 63-75. DOI:10.2307/2411661

Osse, J.W.M. (1990). Form changes in fish larvae in relation to changing demands of function. Netherlands Journal of Zoology, 40, 362-385. DOI:10.1163/156854289X00354

Osse, J.W.M. y van den Boogaart, J.G.M. (1995). Fish larvae, development, allometric growth and the aquatic environment. LK. ICES Marine Science Symposia. 201, 21-34 Retrieved from URL https://wur.on.worldcat.org/oclc/ 1019079684. ICES Mar. Sci. Symp. 201.

Osse, J.W.M., van den Boogaart, J.G.M., van Snik, G.M.J., y van der Sluys, L. (1997). Priorities during early growth of fish larvae. Aquaculture, 155, 249-258. DOI:10.1016/S0044-8486(97)00126-9

Padrós, F., Villalta, M., Gisbert, E., y Estévez, A. (2011). Morphological and histological study of larval development of the Senegal sole Solea senegalensis: an integrative study. Journal of Fish Biology, 79, 3-32. DOI:10.1111/j.1095-8649.2011.02942.x

Pankhurst, N.W., y Montgomery, J.C. (1994). Uncoupling of Visual and Somatic Growth in the Rainbow Trout Oncorhynchus mykiss. Brain, Behavior and Evolution, 44 , 149-155. DOI:10.1159/000113586

Peña, R., y Dumas, S. (2009). Development and allometric growth patterns during early larval stages of the spotted sand bass Paralabrax maculatofasciatus 
(Percoidei: Serranidae). Scientia Marina, 73, 183-189. DOI:10.3989/scimar.2009.73s1183

Portella, M.C., Leitão, N.J., Takata, R., y Lopes, T. (2012). Alimentação e nutrição de larvas. In Nutriaqua: nutrição e alimentação de espécies de interesse para a aquicultura brasileira. (Cap 1. pp,185-216). Florianópolis, Brasil: Sociedade Brasileira de Aquicultura e Biologia Aquática.

Powles, H., y Markle, D.F. (1983). Identification of Larvae. In H.G, Moser, W.J, Richards, D.M, Cohen, M.P, Fahay, A.W, Kendall, Jr., and S.L, Richardson (Eds.). Ontogeny and systematic of fishes (Cap 2. pp, 31-32). La Jolla, Califrornia. USA: American Society of Ichthyologists and Herpetologists

Raposo-Maciel, C.M.R., Teixeira-Lanna, E.A., Maciel-Junior, A., Juarez-Lopes, D., Andrade-Neves, C., y Menin, E. (2010). Morphological and behavioral development of the piracanjuba larvae. Revista Brasilera de Zootecnia, 39, 961-970. DOI:10.1590/S1516-35982010000500004

Reynalte-Tataje, D., Zaniboni-Filho, E., y Esquivel, J. (2004). Embryonic and larvae development of piracanjuba, Brycon orbignyanus Valenciennes, 1849 (Pisces, Characidae). Acta Scientiarum - Biological Sciences. 26, 67-71. DOI:10.4025/actascibiolsci.v26i1.1660

Ricker, W.E. (1979). Growth rates and models. In W. Hoar, D. Randall, J. Brett (Eds.). Fish physiology, bioenergetics and growth (pp. 678-685). New York, USA: Academy press.

Romagosa, E., Narahara, M.Y., y Fenerich-Verani, N. (2001). Stages of embryonic development of the "Matrinxã", Brycon cephalus (Pisces, Characidae). Boletim do Instituto de Pesca, 27, 27-32. DOI:10.1016/j.eurpsy.2016.01.2011

Sampaio-Nakauth, A.C.S., Villacorta-Correa, M.A., Figueiredo, M.R., Bernardino, G., França, J.M. (2016). Embryonic and larval development of Brycon amazonicus (SPIX \& AGASSIZ, 1829). Brazilian Journal of Biology, 109, 109-116. DOI:10.1590/1519-6984.13914

Souza, G., Melo, E.J.T., Caramaschi, E.P., Andrade, D.R., y Monteiro, L.R. (2016). Early development and allometric growth patterns of the grumatã (Prochilodus vimboides Kner, 1859). Zygote, 24, 428-441. DOI:10.1017/S0967199415000350

Souza, G., Caramaschi, E.P., y Monteiro, L.R. (2017). Morphometrics and allometry of the larvae of five characiformes species in the Paraíba do sul river basin. Zygote, 25, 507518. DOI:10.1017/S096719941700034X

Stewart, J. (Ed). (2008). Single variable calculus: Early tracendentals, (6th ed). Belmont, CA. USA: Thomson Learning, Inc.

Sversut de Alexandre, J., Ninhaus-Silveira, A., VerssimoSilveira, R., Buzollo, H., Senhorini, J.A., y PenteadoChaguri, M. (2010). Structural analysis of the embryonic development in Brycon cephalus (Gnther, 1869). Zygote, 18, 173-182. DOI:10.1017/S0967199409990190

Tsai, H.Y., Chang, M., Liu, S.C., Abe, G., y Ota, K.G. (2013). Embryonic development of goldfish (Carassius auratus): a model for the study of evolutionary change in developmental mechanisms by artificial selection. Developmental Dynamics, 242, 1262-1283. DOI:10.1002/dvdy.24022

Van Snik, G.M.J., Van den Boogaart, J.G.M., y Osse, J.W.M. (1997). Larval growth patterns in Cyprinus carpio and
Clarias gariepinus with attention to the finfold. Journal of Fish Biology, 50, 1339-1352. DOI:10.1111/j.10958649.1997.tb01657.x

Vandewalle, P., Germeau, G., Besancenet, P., Parmentier, E., y Baras, E. (2005). Early development of the head skeleton in Brycon moorei (Pisces, Ostariophysi, Characidae). Journal of Fish Biology, 66, 996-1024. DOI:10.1111/j.1095-8649.2005.00656.x

Walpole, R.E.., Myers, R.H., Myers, S.L., y Ye, K. (2012). Probabilidad y estadística para ingeniería y ciencias. (9th. ed). México, DF: Pearson Education, Inc.

Zeferino-Gomes, R., Sato, Y., Rizzo, E., y Bazzoli, N. (2011). Early development of Brycon orthotaenia (Pisces: Characidae). Zygote, 21, 11-20. DOI:10.1017/S0967199411000311 